\title{
The Canopy Graph and Level Statistics for Random Operators on Trees
}

\author{
Michael Aizenman and Simone Warzel \\ Departments of Mathematics and Physics, \\ Princeton University, Princeton NJ 08544, USA.
}

October 29, 2018

\begin{abstract}
For operators with homogeneous disorder, it is generally expected that there is a relation between the spectral characteristics of a random operator in the infinite setup and the distribution of the energy gaps in its finite volume versions, in corresponding energy ranges. Whereas pure point spectrum of the infinite operator goes along with Poisson level statistics, it is expected that purely absolutely continuous spectrum would be associated with gap distributions resembling the corresponding random matrix ensemble. We prove that on regular rooted trees, which exhibit both spectral types, the eigenstate point process has always Poissonian limit. However, we also find that this does not contradict the picture described above if that is carefully interpreted, as the relevant limit of finite trees is not the infinite homogenous tree graph but rather a single-ended "canopy graph". For this tree graph, the random Schrödinger operator is proven here to have only pure-point spectrum at any strength of the disorder. For more general single-ended trees it is shown the spectrum is always singular - pure point possibly with singular continuous component which is proven to occur in some cases.
\end{abstract}

Keywords: Random operators, level statistics, canopy graph, Anderson localization, absolutely continuous spectrum, singular continuous spectrum.

(2000 Mathematics Subject Classifiction: 47B80, 60K40) 


\section{Contents}

1 Introduction 3

1.1 An overview . . . . . . . . . . . . . . . . . 3

1.2 The random operator on finite subgraphs of a regular tree . . . . . . 4

1.3 The canopy operator ................. 6

2 Conditions for Poisson statistics for tree operators 8

2.1 The density bounds of Wegner and Minami . . . . . . . . . . . . . 8

2.2 Proof strategy and a sufficient condition ............ 8

3 Decay estimates of the Green function 12

3.1 The decay of fractional moments . . . . . . . . . . . . . . 13

3.2 Lower bound on the Lyapunov exponent . . . . . . . . . . . . . . . . 16

3.3 Proof of Theorem $3.2 \ldots \ldots \ldots \ldots$

4 Proof of Poisson statistics for tree operators 18

4.1 Divergent fluctuations of the spectral measure in the bulk . . . . . . 18

4.2 The intensity measure . . . . . . . . . . . . . . . . 20

4.3 Proof of Theorem $1.1 \ldots \ldots \ldots 22$

5 Complete localization for random operators on the canopy graph 23

6 The spectra of random operators on single-ended trees 26

6.1 Absence of absolutely continuous spectrum . . . . . . . . . . . 26

6.2 Appearance of singular continuous spectrum . . . . . . . . . 27

A Some basic properties of the canopy operator 31

A.1 Existence of the canopy density of states measure . . . . . . . . . . 31

A.2 Spectrum of the adjacency operator on the canopy graph . . . . . . 33

B Negligibility in probability of the spectral measure within the singular spectrum

C A decorated Delyon-Kunz-Souillard bound 35

C.1 Proof of Proposition 6.2 . . . . . . . . . . . . . . . . 37

D Discussion 38 


\section{Introduction}

\subsection{An overview}

For random operators with extensive disorder it is generally expected that there is an interesting link between the nature of the spectra of the infinite operator and the statistics of energy gaps of the finite-volume restriction of the random operator. Extensively studied examples of operators with disorder include the Schrödinger operator with random potential [CL90, PF92, St01] and the quantum graph operators, as in [KS99, ASW06b]. The often heard conjecture (see eg. [AS86, Sh+93, Ef97, DR03] and references therein) is that on the scale of typical energy spacing the energy levels will exhibit Poisson statistics throughout the pure point (pp) spectral regimes, and level repulsion through energy ranges for which the infinite systems has absolutely continuous (ac) spectrum.

The presence of pp spectra for random Schrödinger operators on $\ell^{2}\left(\mathbb{Z}^{d}\right)$ or $L^{2}\left(\mathbb{R}^{d}\right)$ is now thoroughly investigated. In this context, the conjectured Poisson statistics has been established throughout the localization regime for the lattice cases [Mi96], and also for the $d=1$ continuum operators [Mo81], which exhibit only pure point spectra. For dimensions $d>2$ it is expected that random operators will exhibit also ac spectra. However, so far the only cases of operators with extensive disorder for which the existence of an $a c$ spectral component was proven are operators on tree graphs [K195, K198, ASW06a, FHS07]. Attempting to analyze the conjecture in that context we encountered two surprises, on which we would like to report in this note:

1. For random operators on trees, under an auxiliary technical assumption which is spelled below, the level distribution is given by Poisson statistics through the entire spectral regime. In particular, the statistics of the neighboring levels is typically free of level repulsion even throughout the spectral regimes where the infinite tree operator has $a c$ spectrum.

2. For the purpose of the level statistics of finite tree graph operators, as observed within energy windows scaled by a volume factor, the relevant infinite graph is not the regular tree graph, but another one, which is called here the canopy graph. This graph is isomorphic to the horoball subgraphs of the regular tree, in the terminology explained in [Wo00].

The second point is related to the known result, proven in [Sz89, Sz90, Thm.1.1], concerning the density of states of Schrödinger operators on hyperbolic spaces (see Section 1.3).

The main surprise (1.) is then somewhat reconciled with the above general expectation by the next result:

3. The corresponding random operator on the (infinite) canopy graph, has only pp spectrum at any non-zero level of extensive disorder.

In the above statement, the absence of an absolutely continuous component is readily explained by the fact that the canopy graph has exactly one end - in the sense (see, e. g. [Wo00]) that from each point on it emanates exactly one infinite path. However, 
some more detailed analysis is required to prove that the spectrum is pure point. To make that clear we also prove the following, which may be of some independent interest:

4. There are tree graphs with exactly one end on which the spectrum of the corresponding Schrödinger operator almost surely has a singular continuous spectral component.

We shall now make those statements more explicit.

\subsection{The random operator on finite subgraphs of a regular tree}

Let $\mathcal{T}$ denote the vertex set of a rooted tree graph for which all vertices have $K$ neighbors in directions away from the root 0 , for some fixed $K \geq 2$. Out of the infinite tree $\mathcal{T}$ we carve an increasing sequence of finite trees of depth $L$, denoting:

$$
\mathcal{T}_{L}:=\{x \in \mathcal{T}: \operatorname{dist}(0, x) \leq L\},
$$

Here $\operatorname{dist}(\cdot, \cdot)$ refers to the natural distance between two vertices in $\mathcal{T}$. The adjacency operator on the Hilbert space of square-summable functions $\psi \in \ell^{2}\left(\mathcal{T}_{L}\right)$ is given by

$$
(A \psi)(x):=\sum_{\substack{y \in \mathcal{T}_{L}: \\ \operatorname{dist}(x, y)=1}} \psi(y) .
$$

In the notation for $A$ we omit the index $\left(\mathcal{T}_{L}\right)$ indicating on what $\ell^{2}$-space the operator acts. We will be concerned with random perturbations of the adjacency operator, namely self-adjoint operators of the form

$$
H_{\mathcal{T}_{L}}:=A+V+B
$$

acting in $\ell^{2}\left(\mathcal{T}_{L}\right)$, with $V$ a random potential and $B$ a boundary term, both given by multiplication operators:

$$
\begin{aligned}
& (V \psi)(x):=\omega_{x} \psi(x), \\
& (B \psi)(x):=\left\{\begin{array}{cl}
b \psi(x) & \text { if } \operatorname{dist}(0, x)=L \\
0 & \text { otherwise } .
\end{array}\right.
\end{aligned}
$$

Here $\left\{\omega_{x}\right\}_{x \in \mathcal{T}}$ stands for a collection of independent identically distributed (iid) random variables, and $b \in \mathbb{R}$ is a fixed number. The latter serves as a control parameter, in effect allowing to vary the boundary conditions at the outer boundary, a term by which we refer to the set $\partial \mathcal{T}_{L}:=\{x \in \mathcal{T}: \operatorname{dist}(0, x)=L\}$.

Throughout this discussion we restrict ourselves to random potentials whose probability distribution meets the following condition:

Assumption A1: The distribution of the potential variables $\omega_{x}$ is of bounded density, $\varrho \in L^{\infty}(\mathbb{R})$, and satisfies $\int_{\mathbb{R}}\left|\omega_{0}\right|^{\tau} \varrho\left(\omega_{0}\right) d \omega_{0}<\infty$ for some $\tau>0$. 
The main object of interest will be the random point process of eigenvalues of $H_{\mathcal{T}_{L}}$, seen on the scale of the mean level spacing. For a finite operator, the expected number of eigenvalues in an interval is proportional to the number of sites of the finite graph, $\left|\mathcal{T}_{L}\right|$ (see the Wegner estimate (2.1) below). It is therefore natural to consider the point process of the eigenvalues as seen under the magnification by the volume. Thus, for a given energy $E \in \mathbb{R}$ we consider the random point measure

$$
\mu_{L}^{E}:=\sum_{n} \delta_{\left|\mathcal{T}_{L}\right|\left(E_{n}\left(\mathcal{T}_{L}\right)-E\right)},
$$

where $\left\{E_{n}\left(\mathcal{T}_{L}\right)\right\}$ denotes the sequence of random eigenvalues of $H_{\mathcal{T}_{L}}$, counting multiplicity.

Our main results are derived under the additional assumption:

Assumption A2: The expectation values $\mathbb{E}\left[\ln \left|\left\langle\delta_{0},\left(H_{\mathcal{T}_{L}}-E\right)^{-1} \delta_{0}\right\rangle\right|\right]$ are equicontinuous functions of $E \in I$ over some Borel set $I \subset \mathbb{R}$.

An explicit example, which satisfies both Assumptions $\mathbf{A 1}$ and $\mathbf{A 2}$ for $I=\mathbb{R}$, is the Cauchy distribution. In that case, Cauchy integration allows to equate the above expectation value with the resolvent at an energy off the real axis, and then $\mathbf{A 2}$ is easily seen to be valid. For the general case, through a Thouless-type formula one gets

$$
\mathbb{E}\left[\ln \left|\left\langle\delta_{0},\left(H_{\mathcal{T}_{L}}-z\right)^{-1} \delta_{0}\right\rangle\right|\right]=\operatorname{Re} \int_{\mathbb{R}} \frac{\nu_{L}(E)}{E-z} d E
$$

where $\nu_{L}(E):=\mathbb{E}\left[\operatorname{Tr} P_{(-\infty, E)}\left(H_{\mathcal{T}_{L}}\right)\right]-K \mathbb{E}\left[\operatorname{Tr} P_{(-\infty, E)}\left(H_{\mathcal{T}_{L-1}}\right)\right]$ defines the spectral shift function related to the removal of the root in $\mathcal{T}_{L}$. The symbol $\operatorname{Tr}$ refers to the trace and $P_{I}$ denotes the spectral projection onto the Borel set $I \subset \mathbb{R}$. Assumption A2 is therefore connected to the regularity of this spectral shift function. Such regularity may be deduced from some of the results in [AK92] which address distributions "near" the Cauchy case.

The main result of the present paper is

Theorem 1.1 (Poisson statistics). Let $H$ be a random Schrödinger operator $H$, as in (1.3), for which the conditions $\mathbf{A 1}$ and $\mathbf{A 2}$ hold for some interval $I \subset \mathbb{R}$. Then for Lebesgue almost every $E \in I$ the random point measures $\mu_{L}^{E}$ converges to a Poisson point measure $\mu^{E}$ as $L \rightarrow \infty$.

The intensity of the limiting Poisson point process $\mu^{E}$ is given by the Lebesgue measure times the canopy density of states $d_{\mathcal{C}}(E)$, which is the the topic of Subsection 4.2 In particular, it is shown there that this intensity is non-zero in some energy regimes. The proof of Theorem 1.1 is provided in Section 4 below. The convergence refers to the usual notion of weak convergence of random point measures [Ka02].

As explained above, at first glance Theorem 1.1 may appear to be surprising, since it is known that random Schrödinger operators on regular infinite trees exhibit also spectral regimes where the spectrum is ac [K195, K198, ASW06a, FHS07]. Furthermore, 
the cases for which this result was established include some for which both assumptions are satisfied, and the ac spectrum was even shown to be pure in the present setting [K198]. Thus, the result may appear to fly in the face of the oft repeated expectation that ac spectra of the infinite volume limit should be linked with level repulsion of the finite subsystems. However, that discrepancy is resolved by the observations presented next.

\subsection{The canopy operator}

It may seem natural to take the line that the infinite-volume limit of the sequence of finite trees $\mathcal{T}_{L}$ is the infinite tree $\mathcal{T}$. That is indeed what the graph converges to when viewed from the perspective of the root, or from any site at fixed distance from the root. However, if one fixes the perspective to be that of a site at the outer boundary of $\mathcal{T}_{L}$, the limit which emerges is different. We use the term canopy tree to describe that limiting graph. More explicitly, the rooted canopy tree $\mathcal{C}$ is recursively defined in terms of a hierarchy of infinite layers of vertices: starting from an infinite outermost boundary layer, $\partial \mathcal{C}$, each layer is partitioned into sets of $K$ elements, and the elements of each component are joined to a common site in the next layer; see Figure 1.

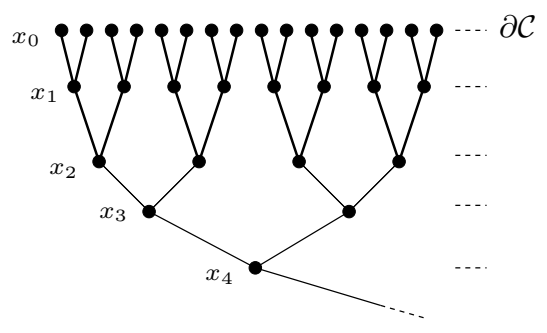

Figure 1: The canopy graph $\mathcal{C}$ for $K=2$. The dots indicate that the boundary layer $\partial \mathcal{C}$ as well as any layer below is infinite. The vertices $x_{0}, x_{1}, x_{2}, \ldots$ mark the points on the unique path $\mathcal{P}\left(x_{0}\right)$ of $x_{0}$ to "infinity".

Two remarks apply:

1. The canopy graph can be imbedded in the regular tree. It is isomorphic to a horoball, the canopy's outermost boundary layer corresponding to a horosphere and its different layers to horocycles - in the terminology explained, e.g, in [Wo00].

2. The observation that a given nested sequence of graphs may have different limits applies also to other graphs. In particular, for the sequence $[-L, L]^{d} \cap \mathbb{Z}^{d}$ analogs of the canopy construction yield the graphs $\mathbb{N} \times \mathbb{Z}^{(d-1)}$, and also $\mathbb{N}^{k} \times \mathbb{Z}^{(d-k)}$ for any $0 \leq k \leq d$.

In view of the multiplicity of the limiting graphs, one may ponder which of them is of relevance for a given question. If the question concerns an extensive quantity, e.g. $\operatorname{Tr} F\left(H_{L}\right)=\sum_{x}\left\langle\delta_{x}, F\left(H_{L}\right) \delta_{x}\right\rangle$, where $H_{L}$ is a finite-range operator and $F$ some smooth function, then the answer depends on how the environment appears from the perspective of a point which is chosen at random uniformly within the finite graph. In this respect there is a fundamental difference between the finite cubic subgraphs of $\mathbb{Z}^{d},[-L, \ldots, L]^{d}$, and the finite subgraphs of a regular tree. In the former case, for $L \rightarrow \infty$, under the uniform sampling the distance from the boundary regresses to 
infinity, and $\mathbb{Z}^{d}$ is the natural limit. However, for the tree graphs $\mathcal{T}_{L}$ the distribution of the distance to the boundary converges to the exponential distribution: the fraction of points whose distance to the outer boundary exceeds $n$, decays as $K^{-n}$. In this case it is the canopy graph which captures the limit. For an explicit formulation of the statement we introduce the canopy operator acting on $\ell^{2}(\mathcal{C})$,

$$
H_{\mathcal{C}}:=A+V+B
$$

Here, $A$ is the adjacency operator on $\ell^{2}(\mathcal{C})$ which is defined similarly to 1.2 , and $B$ is a boundary term acting as in 1.5 , with the same $b \in \mathbb{R}$. Moreover, the iid random variables $\left\{\omega_{x}\right\}_{x \in \mathcal{C}}$ underlying the random multiplication operator $V$ are supposed to satisfy A1.

Associated to $H_{\mathcal{C}}$ is the following density of states (dos) measure given by

$$
n_{\mathcal{C}}(I):=\frac{K-1}{K} \sum_{n=0}^{\infty} K^{-n} \mathbb{E}\left[\left\langle\delta_{x_{n}}, P_{I}\left(H_{\mathcal{C}}\right) \delta_{x_{n}}\right\rangle\right]
$$

where the sum ranges over all vertices $x_{0}, x_{1}, \ldots$ on the unique path $\mathcal{P}\left(x_{0}\right)$ of a given vertex $x_{0} \in \partial \mathcal{C}$ to infinity, see Figure 1 Note that $n_{\mathcal{C}}$ does depend on the choice of the boundary conditions parameter $b \in \mathbb{R}$, however it is independent of the choice of $x_{0} \in \partial \mathcal{C}$ on the boundary.

Theorem 1.2. The density of states of the finite tree $\mathcal{T}_{L}$ is asymptotically given by $n_{\mathcal{C}}$ in the sense that, with probability one: for any bounded continuous $F \in C_{b}(\mathbb{R})$

$$
\lim _{L \rightarrow \infty}\left|\mathcal{T}_{L}\right|^{-1} \operatorname{Tr} F\left(H_{\mathcal{T}_{L}}\right)=\int_{\mathbb{R}} n_{\mathcal{C}}(d E) F(E) .
$$

The statement reflects the fact that on trees, asymptotically, almost all points are located not far from the surface. The proof is given in Subsection A.1. The fact that for non-amenable graphs like trees bulk averages as in 1.10 do not converge to corresponding infinite-volume quantities is well known. In particular, a continuum analogue of Theorem 1.2 was presented in [Sz89, Sz90, Thm.1.1] where it is shown that the finite-volume density of states (dos) of a Laplacian plus Poissonian random potential on hyperbolic space converges to the dos on a horoball. Analogous statements apply to the dos of periodic Schrödinger operators on hyperbolic spaces [AS93].

Part of the suprise of Theorem 1.1 is now removed by the following result, which is proven in Section 5 .

Theorem 1.3 (Localization of canopy states). If the conditions $\mathbf{A 1}$ and $\mathbf{A 2}$ hold for $I \subset$ $\mathbb{R}$, then the random canopy operator $H_{\mathcal{C}}$ has almost surely only pure point spectrum in $I$.

It may also be of interest to note the following curious property of $\mathcal{C}$.

Theorem 1.4 (Spectrum of the adjacency operator). The spectrum of of the adjacency operator with (constant) boundary conditions, $A+B$ on $\ell^{2}(\mathcal{C})$, is only pure point with compactly supported eigenfunctions. 
A more detailed description of the spectrum of the adjacency operator can be found in Subsection A.2 The considerations yielding Theorem 1.4 are essentially as in the analysis of the regular tree in [AF00].

We postpone further comments on possible directions for studies of the relation which was explored in this work to the concluding section, Sect.D

\section{Conditions for Poisson statistics for tree operators}

\subsection{The density bounds of Wegner and Minami}

Key information on the point process which describes the eigenvalues of the random operator as seen under the magnification by the volume factor $\left|\mathcal{T}_{L}\right|$ is provided in the following two essential estimates. The Wegner estimate implies that the mean density of states is bounded relative to the Lebesgue measure. The Minami bound guarantees that the energy levels are non-degenerate on the scale of the mean level spacing.

Proposition 2.1. Under the assumption $\mathbf{A 1}$, for every bounded Borel set $I \subset \mathbb{R}$ and every $L \in \mathbb{N}$

$$
\mathbb{P}\left(\operatorname{Tr} P_{I}\left(H_{\mathcal{T}_{L}}\right) \geq 1\right) \leq \mathbb{E}\left[\operatorname{Tr} P_{I}\left(H_{\mathcal{T}_{L}}\right)\right] \leq|I|\left|\mathcal{T}_{L}\right|\|\varrho\|_{\infty}
$$

(the Wegner estimate), and

$$
\begin{aligned}
\sum_{m=2}^{\infty} \mathbb{P}\left(\operatorname{Tr} P_{I}\left(H_{\mathcal{T}_{L}}\right) \geq m\right) & \leq \mathbb{E}\left[\operatorname{Tr} P_{I}\left(H_{\mathcal{T}_{L}}\right)\left(\operatorname{Tr} P_{I}\left(H_{\mathcal{T}_{L}}\right)-1\right)\right] \\
& \leq \pi^{2}|I|^{2}\left|\mathcal{T}_{L}\right|^{2}\|\varrho\|_{\infty}^{2}
\end{aligned}
$$

(the Minami estimate). Here $\operatorname{Tr} P_{I}\left(H_{\mathcal{T}_{L}}\right)$ stands for the trace of the spectral projection of $H_{\mathcal{T}_{L}}$ onto $I$.

A proof of the Wegner bound (2.1) can be found in [We81, [PF92]. Minami's estimate (2.2) is presented in [Mi96, Lemma 2, Eq. (2.48)], see also [GV06]. Although it is stated there for $\mathbb{Z}^{d}$ only, its derivation clearly applies to all graphs.

\subsection{Proof strategy and a sufficient condition}

The main effort in the proof of the convergence of the energy level process to a Poisson limit is to establish infinite divisibility. In the background are the following observations.

1. The Wegner estimate 2.1 implies tightness of the collection of random variables $\left\{\mu_{L}^{E}(I)\right\}$ for all intervals $I \subset \mathbb{R}$ and every $E \in \mathbb{R}$. This in turn guarantees that for every $E \in \mathbb{R}$ the sequence of measures $\left\{\mu_{L}^{E}\right\}$, is tight with respect to the vague topology on the space of Borel measures on the real line. Since the subspace of point measures is closed with respect to this topology, all accumulation points of the above sequence are point measures [Ka02]. 
2. In order to show that any accumulation point is a Poisson measure, it is sufficient to prove that each such point is infinitely divisible and almost surely has no double points. The latter is guarantied already by the Minami estimate.

3. Once the divisibility property is established, for convergence of the point process it suffices to show that the intensity measure of any accumulation point is given by some common measure $([\overline{\mathrm{Ka} 02]})$. In our case, that measure is the canopy mean density of states $n_{\mathcal{C}}$.

Turning to the divisibility, one may note that for random operators on $\ell^{2}\left(\mathbb{Z}^{d}\right)$ the divisibility and convergence of the energy level process to a Poisson process were proven by Minami under a natural localization condition (the fractional moment characterization of the pp spectral regime [Mi96]). However, Minami's proof does not extend to tree graphs, since it makes use of the fact that cubic regions in $\mathbb{Z}^{d}$ have the van Hove property, which is that most of the volume is, asymptotically, far from the surface. While this approach does not apply to trees, or hyperbolic spaces, with positive Cheeger isoperimetric constant, for trees there is another pathway towards infinite divisibility of any accumulation point of $\left\{\mu_{L}^{E}\right\}$. In order to show $K^{N}$-divisibility at some arbitrary $N \in \mathbb{N}$, we cut the finite tree $\mathcal{T}_{L}$ below the $N$ th generation. This leaves us with a "tree stump" and the subtrees $\mathcal{T}_{L}(x)$ which are forward to vertices $x$ in the $N$ th generation. Associated with the above collection of forward subtrees is the collection of iid point measures

$$
\mu_{x, L}^{E}:=\sum_{n} \delta_{\left|\mathcal{T}_{L}\right|\left(E_{n}\left(\mathcal{T}_{L}(x)\right)-E\right)} .
$$

For the sum $\sum_{\operatorname{dist}(0, x)=N} \mu_{x, L}^{E}$ to be asymptotically equal to $\mu_{L}^{E}$ as $L \rightarrow \infty$, so that any of its accumulation points is $K^{N}$-divisible, it suffices that the spectral measures associated with the roots of the subtrees satisfy the following fluctuation condition.

For any site $x \in \mathcal{T}_{L}$ the spectral measure is defined for Borel sets $I \subset \mathbb{R}$ by

$$
\sigma_{x, L}(I):=\left\langle\delta_{x}, P_{I}\left(H_{\mathcal{T}_{L}}\right) \delta_{x}\right\rangle .
$$

By a Wegner-type estimate the averaged spectral measure, $\mathbb{E}\left[\sigma_{x, L}\right]$, is seen to be ac with a density bounded uniformly in $L \in \mathbb{N}$. The condition we require for the proof of divisibility is that the typical value of $\sigma_{x, L}$ on the scale of its mean, $\left|\mathcal{T}_{L}\right|^{-1}$, is much smaller than the average value. More explicitly:

Definition 2.1. For a fixed site $x \in \mathcal{T}$ and energy $E \in \mathbb{R}$, the sequence of spectral measures $\left\{\sigma_{x, L}\right\}$ is said to be negligible in probability iff for all $w>0$

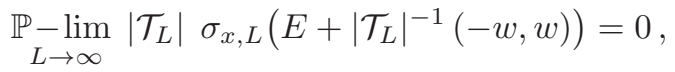

where the limit refers to distributional convergence.

Several remarks apply:

1. The prelimit quantity in 2.5) compares the spectral measure $\sigma_{x, L}$ to the blownup Lebesgue measure of the corresponding interval. In terms of the normalized 
eigenfunctions $\psi_{n}\left(\mathcal{T}_{L}\right) \in \ell^{2}\left(\mathcal{T}_{L}\right)$ of $H_{\mathcal{T}_{L}}$, with the corresponding eigenvalues $E_{n}\left(\mathcal{T}_{L}\right)$, one has:

$$
\sigma_{x, L}(I)=\sum_{E_{n}\left(\mathcal{T}_{L}\right) \in I}\left|\left\langle\delta_{x}, \psi_{n}\left(\mathcal{T}_{L}\right)\right\rangle\right|^{2}
$$

By Wegner's estimate (2.1) the mean number of levels within any given Borel set with Lebesgue measure proportional to $\left|\mathcal{T}_{L}\right|^{-1}$ is bounded uniformly in $L$. If the corresponding eigenfuctions are spread uniformly over the volume, and the relevant spectral density is non-zero, then the above condition is not satisfied, since whenever an eigenvalue falls within the interval the rescaled spectral measure is not smaller than order 1 . Thus, condition (2.5) is equivalent to the statement that either $i$. the probability of finding an eigenvalue in the energy window vanishes, or $i i$. the corresponding eigenfunctions are spread very unevenly over the volume, so that typically $\left|\left\langle\delta_{x}, \psi_{n}\left(\mathcal{T}_{L}\right)\right\rangle\right|^{2} \times\left|\mathcal{T}_{L}\right|<<1$ even though the average of the quantity over the volume (or over $n$ ) is 1 . In other words, on the scale of their mean the eigenfunctions exhibit divergent fluctuations.

2. We shall show below, in Appendix $B$ that the above scenario $i$. occurs if for some energy range the (weak) limiting measure, $\left\langle\delta_{x}, P .\left(H_{\mathcal{T}}\right) \delta_{x}\right\rangle=\lim _{L \rightarrow \infty} \sigma_{x, L}$ for $x \in \mathcal{T}$, is purely singular. In that case, due to the mutual singularity, the spectral measures underperforms at Lebesgue - chosen $E \in \mathbb{R}$, in small intervals of arbitrary scale. It is more of an issue to verify that 2.5) holds throughout the regime of ac spectrum of $H_{\mathcal{T}}$. This will be proven in Subsection 4.1 below, where we show that for canopy graph scenario $i$ i. is in effect.

The criterion for Poisson statistics may now be formulated as follows.

Theorem 2.2 (Condition for Poisson statistics). Suppose that the sequence of spectral measures at the root, $\left\{\sigma_{0, L}\right\}$, is negligible in probability at $E \in \mathbb{R}$. Then for any $N \in \mathbb{N}$ the sum $\sum_{\operatorname{dist}(0, x)=N} \mu_{x, L}^{E}$ converges weakly to the same limit as $\mu_{L}^{E}$, i.e., for all $\psi \in L_{+}^{1}(\mathbb{R})$

$$
\lim _{L \rightarrow \infty}\left|\mathbb{E}\left[e^{-\sum_{\operatorname{dist}(0, x)=N} \mu_{x, L}^{E}(\psi)}\right]-\mathbb{E}\left[e^{-\mu_{L}^{E}(\psi)}\right]\right|=0 .
$$

As a consequence, all accumulation points of $\mu_{L}^{E}$ are random Poisson measures.

Proof. Since the set of functions $\varphi_{z}:=\operatorname{Im}(\cdot-z)^{-1}$ with $z \in \mathbb{C}^{+}$are dense in $L_{+}^{1}(\mathbb{R})$, it suffices to verify 2.7) for such functions. It is easy to see that the latter follows from the distributional convergence

$$
\underset{L \rightarrow \infty}{\mathbb{P}-\lim _{\operatorname{dist}(0, x)=N}}\left|\mu_{x, L}^{E}\left(\varphi_{z}\right)-\mu_{L}^{E}\left(\varphi_{z}\right)\right|=0 .
$$


Abbreviating $\xi_{L}:=E+z\left|\mathcal{T}_{L}\right|^{-1}$ the prelimit in (2.8) can be written as

$$
\begin{aligned}
& \frac{1}{\left|\mathcal{T}_{L}\right|}\left|\sum_{\operatorname{dist}(0, x)=N} \operatorname{Im} \operatorname{Tr}\left(H_{\mathcal{T}_{L}(x)}-\xi_{L}\right)^{-1}-\operatorname{Im} \operatorname{Tr}\left(H_{\mathcal{T}_{L}}-\xi_{L}\right)^{-1}\right| \\
& \leq \frac{1}{\left|\mathcal{T}_{L}\right|} \sum_{|y|<N} \operatorname{Im}\left\langle\delta_{y},\left(H_{\mathcal{T}_{L}}-\xi_{L}\right)^{-1} \delta_{y}\right\rangle \\
& +\frac{1}{\left|\mathcal{T}_{L}\right|} \sum_{\operatorname{dist}(0, x)=N}\left|\sum_{y \in \mathcal{T}_{L}(x)}\left\langle\delta_{y},\left(H_{\mathcal{T}_{L}(x)}-\xi_{L}\right)^{-1} \delta_{y}\right\rangle-\left\langle\delta_{y},\left(H_{\mathcal{T}_{L}}-\xi_{L}\right)^{-1} \delta_{y}\right\rangle\right| .
\end{aligned}
$$

The first term on the right side converges to zero in distribution as $L \rightarrow \infty$. Using the resolvent identity twice the modulus in the second term is seen to be equal to

$$
\begin{gathered}
\left|\sum_{y \in \mathcal{T}_{L}(x)}\left\langle\delta_{y},\left(H_{\mathcal{T}_{L}(x)}-\xi_{L}\right)^{-1} \delta_{x}\right\rangle\left\langle\delta_{x^{-}}\left(H_{\mathcal{T}_{L}}-\xi_{L}\right)^{-1} \delta_{x^{-}}\right\rangle\left\langle\delta_{x},\left(H_{\mathcal{T}_{L}(x)}-\xi_{L}\right)^{-1} \delta_{y}\right\rangle\right| \\
\leq\left\langle\delta_{x},\left|H_{\mathcal{T}_{L}(x)}-\xi_{L}\right|^{-2} \delta_{x}\right\rangle\left|\left\langle\delta_{x^{-}}\left(H_{\mathcal{T}_{L}}-\xi_{L}\right)^{-1} \delta_{x^{-}}\right\rangle\right|, \quad \text { (2.10) }
\end{gathered}
$$

where $x^{-}$is the backward neighbor of $x$ in $\mathcal{T}_{L}$. The second term in 2.10 is bounded in probability as $L \rightarrow \infty$ as is seen from the fractional moment bound (3.1) below. Thanks the fluctuation condition and Lemma 2.3 below the first term, when dividing by $\left|\mathcal{T}_{L}\right|$, converges to zero in this limit.

The previous proof was based on the following

Lemma 2.3. Either of the following is equivalent to the statement that the sequence of spectral measures $\left\{\sigma_{x, L}\right\}$ is negligible in probability, at $E \in \mathbb{R}$ :

1. For all $\alpha \in \mathbb{R}: \quad \underset{L \rightarrow \infty}{\mathbb{P}-\lim _{L}}\left|\mathcal{T}_{L}\right|^{-1}\left\langle\delta_{x},\left(H_{\mathcal{T}_{L}}-E-\alpha\left|\mathcal{T}_{L}\right|^{-1}\right)^{-2} \delta_{x}\right\rangle=0$.

2. For all $z \in \mathbb{C}^{+}: \quad \underset{L \rightarrow \infty}{\mathbb{P}-\lim } \operatorname{Im}\left\langle\delta_{x},\left(H_{\mathcal{T}_{L}}-E-z\left|\mathcal{T}_{L}\right|^{-1}\right)^{-1} \delta_{x}\right\rangle=0$.

Proof. $1 . \Rightarrow 2$. $\Rightarrow$ 'negligibility in probability': These two implications are a consequence of the following chain of elementary inequalities

$$
\begin{aligned}
2(\operatorname{Im} z)^{-1}\left\langle\delta_{x}, P_{I(\operatorname{Re} z, \operatorname{Im} z)}\left(H_{\mathcal{T}_{L}}\right), \delta_{x}\right\rangle & \leq \operatorname{Im}\left\langle\delta_{x},\left(H_{\mathcal{T}_{L}}-z\right)^{-1} \delta_{x}\right\rangle \\
& \leq \operatorname{Im} z\left\langle\delta_{x},\left(H_{\mathcal{T}_{L}}-\operatorname{Re} z\right)^{-2} \delta_{x}\right\rangle,
\end{aligned}
$$

valid for all $z \in \mathbb{C}^{+}$, where $I(E, w):=E+(-w, w)$ denotes the open interval centred at $E$ of width $2 w>0$.

'Negligibility in probability' $\Rightarrow 1$.: We split the prelimit in 1 . into two terms by inserting a spectral projection onto the interval $I_{L}:=I\left(E, w\left|\mathcal{T}_{L}\right|^{-1}\right)$ and its complement. Abbreviating $\xi_{L}:=E+\alpha\left|\mathcal{T}_{L}\right|^{-1}$ the first term is then estimated as follows

$$
\left|\mathcal{T}_{L}\right|^{-1}\left\langle\delta_{x},\left(H_{\mathcal{T}_{L}}-\xi_{L}\right)^{-2} P_{I_{L}}\left(H_{\mathcal{T}_{L}}\right) \delta_{x}\right\rangle \leq\left|\mathcal{T}_{L}\right|^{-1} \frac{\left\langle\delta_{x}, P_{I_{L}}\left(H_{\mathcal{T}_{L}}\right) \delta_{x}\right\rangle}{\operatorname{dist}\left(\sigma\left(H_{\mathcal{T}_{L}}\right), \xi_{L}\right)^{2}}
$$


Using Wegner's estimate (2.1) and (2.5), this term is seen to converge in distribution to zero as $L \rightarrow \infty$ for any $w>0$. The remaining second term is

$$
\begin{aligned}
\left|\mathcal{T}_{L}\right|^{-1}\left\langle\delta_{x},\left(H_{\mathcal{T}_{L}}-\xi_{L}\right)^{-2} P_{I_{L}^{c}}\left(H_{\mathcal{T}_{L}}\right) \delta_{x}\right\rangle \\
\leq 2 w^{-1} \operatorname{Im}\left\langle\delta_{x},\left(H_{\mathcal{T}_{L}}-\xi_{L}+i w\left|\mathcal{T}_{L}\right|^{-1}\right)^{-1} \delta_{x}\right\rangle .
\end{aligned}
$$

The imaginary part of the resolvent is bounded in probability. Therefore the probability that the right side in 2.13) is greater than any arbitrarily small constant is arbitrarily small for $w$ large enough.

\section{Decay estimates of the Green function}

As was shown in [AM93, Thm. II.1], fractional moments of the Green function of rather general random operators are uniformly bounded.

Proposition 3.1 (Fractional moment bounds). Under the assumption A1, for any $s \in$ $(0,1)$,

$$
C_{s}:=\sup _{z \in \mathbb{C}} \sup _{L \in \mathbb{N}} \sup _{x, y \in \mathcal{T}_{L}} \mathbb{E}\left[\left|\left\langle\delta_{x},\left(H_{\mathcal{T}_{L}}-z\right)^{-1} \delta_{y}\right\rangle\right|^{s} \mid\{x, y\}^{c}\right]<\infty,
$$

where the average $\mathbb{E}\left[\cdot \mid\{x, y\}^{c}\right]$ is the conditional expectation with respect to the sigma-algebra the generated by $\left\{\omega_{v}\right\}_{v \in \mathcal{T} \backslash\{x, y\}}$.

The main aim of this section is to prove that fractional moments of the Green function of $H_{\mathcal{T}_{L}}$ are not only bounded but decay exponentially along any ray in the tree.

Theorem 3.2 (Exponential decay I). Assume $\mathbf{A 1}$ and $\mathbf{A 2}$ holds for a bounded Borel set $I \subset \mathbb{R}$. Then there exists $s \in(0,1), \delta, C \in(0, \infty)$ such that for all $E \in I, L \in \mathbb{N}$ and all $x \in \mathcal{T}_{L}$ which are in the future of $y \in \mathcal{T}_{L}$

$$
\mathbb{E}\left[\left|\left\langle\delta_{y},\left(H_{\mathcal{T}_{L}}-E\right)^{-1} \delta_{x}\right\rangle\right|^{s}\right] \leq C \exp [-s(\delta+\ln \sqrt{K}) \operatorname{dist}(x, y)] .
$$

Several remarks apply:

1. Unlike on $\mathbb{Z}^{d}$, for trees the exponential decay (3.2) does not imply complete localization, i.e. dense pure point spectrum at all energies. In fact, the infinitevolume operator $H_{\mathcal{T}}$ has a regime with delocalized eigenstates [K195, K198, ASW06a, FHS07.

2. The rate of decay in 3.2 is related to a Lyapunov exponent of the infinitevolume operator $H_{\mathcal{T}}$, cf. Subsection 3.2 below. Note that in the unperturbed case where $H_{\mathcal{T}}=A$, the decay rate in $(3.2)$ would be given by $\ln \sqrt{K}$. It is important for us that the decay rate in $(3.2)$ is strictly larger. 


\subsection{The decay of fractional moments}

Our proof of Theorem 3.2 is based on reasonings similar to that which applies in in the one-dimensional setup, the reason being that any pair of sites on the tree is connected through a single path. As in one dimension, the Green function decays exponentially at a rate characterized by a Lyapunov exponent. In order to relate the decay rate of the fractional-moment of the Green function to that exponent, the following simple lemma will be of help.

Lemma 3.3. Let $\left(\xi_{j}\right)_{j=1}^{N}$ be a collection of independent, positive random variables with $c:=\max _{j} \mathbb{E}\left[\left(\ln \xi_{j}\right)^{2}\left(\xi_{j}+1\right)\right] / 2<\infty$. Then $X:=\prod_{j=1}^{N} \xi_{j}$ satisfies

$$
\mathbb{E}[X] \leq \exp (\mathbb{E}[\ln X]+N c)
$$

Proof. This is a straightforward consequence of the assumed independence and the elementary inequalities $e^{\alpha} \leq 1+\alpha+\alpha^{2}\left(e^{\alpha}+1\right) / 2$ and $1+\beta \leq e^{\beta}$ valid for all $\alpha$, $\beta \in \mathbb{R}$.

Lemma 3.4. Let $I \subset \mathbb{R}$ be a bounded Borel set and assume A1. Then for every $\varepsilon>0$ there exists $s_{\varepsilon} \in(0,1 / 2)$ and $L_{\varepsilon} \in \mathbb{N}$ such that for all $s \in\left(0, s_{\varepsilon}\right), E \in I, L \geq L_{\varepsilon}$ and $x \in \mathcal{T}_{L}$ with $\operatorname{dist}(0, x) \geq L_{\varepsilon}$

$$
\begin{aligned}
\ln \mathbb{E}\left[\left|\left\langle\delta_{0},\left(H_{\mathcal{T}_{L}}-E\right)^{-1} \delta_{x}\right\rangle\right|^{s}\right] & \\
& \leq \varepsilon \operatorname{dist}(0, x)+s \mathbb{E}\left[\ln \left|\left\langle\delta_{0},\left(H_{\mathcal{T}_{L}}-E\right)^{-1} \delta_{x}\right\rangle\right|\right]
\end{aligned}
$$

The proof is based on the factorization of the Green function on a tree, which we recall from [K198, Eq. (2.8)],

$$
\begin{aligned}
& \left\langle\delta_{0},\left(H_{\mathcal{T}_{L}}-E\right)^{-1} \delta_{x}\right\rangle=\prod_{j=0}^{\operatorname{dist}(0, x)} \Gamma_{j, L} \\
& \quad \text { with } \quad \Gamma_{j, L}:=\left\langle\delta_{x_{j}},\left(H_{\mathcal{T}_{L}\left(x_{j}\right)}-E\right)^{-1} \delta_{x_{j}}\right\rangle .
\end{aligned}
$$

Here $0=: x_{0}, x_{1}, \ldots, x_{\operatorname{dist}(0, x)}:=x$ are the vertices on the unique path connecting the root 0 with $x$. Moreover, $\mathcal{T}_{L}\left(x_{j}\right)$ is that subtree of $\mathcal{T}_{L}$ which is rooted at and forward to $x_{j}$.

Proof of Lemma 3.4 The idea is to group together subproducts of (3.5) und use certain independence properties in order to apply Lemma 3.3. To do so we pick $L_{0} \in \mathbb{N} \backslash\{1\}$ and express the distance of $x$ to the root modulo $L_{0}$,

$$
\operatorname{dist}(0, x)=N_{x} L_{0}+L_{x}
$$


with suitable $N_{x} \in \mathbb{N}_{0}$ and $L_{x} \in\left\{0, \ldots, L_{0}-1\right\}$. Thanks to the factorization (3.5) we may thus write

$$
\begin{aligned}
&\left|\left\langle\delta_{0},\left(H_{\mathcal{T}_{L}}-E\right)^{-1} \delta_{x}\right\rangle\right|^{s}=\left(\prod_{k=0}^{N_{x}-1} X_{k} Y_{k}\right) R \\
& \text { with } \quad X_{k} Y_{k}:=\prod_{j=k L_{0}}^{(k+1) L_{0}-1}\left|\Gamma_{j, L}\right|^{s}, \quad R:=\prod_{j=N_{x} L_{0}}^{\operatorname{dist}(0,|x|)}\left|\Gamma_{j, L}\right|^{s} .
\end{aligned}
$$

Each product $X_{k} Y_{k}$ may now be split into two terms by setting $Y_{k}$ equal to the modulus of a diagonal element of the operator corresponding to the forward subtree $\mathcal{T}_{L}\left(x_{k L_{0}}\right)$,

$$
Y_{k}:=\left|\left\langle\delta_{x_{(k+1) L_{0}-1}},\left(H_{\mathcal{T}_{L}\left(x_{k L_{0}}\right)}-E\right)^{-1} \delta_{x_{(k+1) L_{0}-1}}\right\rangle\right|^{s} .
$$

The point is that in this way we obtain a collection $\left(X_{k}\right)_{k=0}^{N_{x}-1}$ of independent, positive random variables. Moreover,

1. each random variable $X_{k}$ is independent of the value of the potential at vertex $x_{j}$ with $j=(k+1) L_{0}-1$ for some $k \in\left\{0, \ldots, N_{x}-1\right\}$ or $N_{x} L_{0} \leq j \leq$ $\operatorname{dist}(0,|x|)$.

2. the random variable $Y_{k}$ is independent of the value of the potential at vertex $x_{j}$ with $0 \leq j<k L_{0}$.

One may therefore succesively integrate the product in $3.7 \mathrm{~b}$ by first conditioning on the potential at $x_{L_{0}-1}$ thereby integrating $Y_{0}$, then conditioning on $x_{2 L_{0}-1}$ thereby integrating $Y_{1}$ and so forth until we reach $x_{N_{x} L_{0}-1}$ and integrate $Y_{N_{x}-1}$. Thanks to (3.1) these integrals are all uniformly bounded,

$$
\mathbb{E}_{x_{(k+1) L_{0}-1}}\left[Y_{k}\right] \leq C_{s} .
$$

Moreover, conditioning on the values of the potential at $x_{N_{x} L_{0}}$ and $x$, the fractionalmoment bound (3.1) also yields

$$
\mathbb{E}_{x_{N_{x} L_{0}}, x}[R] \leq C_{s} .
$$

One is then left with the integral of the product $\prod_{k=0}^{N_{x}-1} X_{k}$, which can be bounded with the help of Lemma 3.3 Its assumption is satisfied since

$$
\begin{aligned}
2 c:=\max _{k} \mathbb{E}\left[\left(\ln X_{k}\right)^{2}\left(X_{k}+1\right)\right] \\
\leq\left(s L_{0} \max _{j}\left(\mathbb{E}\left[\left(\ln \left|\Gamma_{j, L}\right|\right)^{4}\right]\right)^{1 / 4}+\left(\mathbb{E}\left[\left(\ln Y_{k}\right)^{4}\right]\right)^{1 / 4}\right)^{2} \\
\quad \times\left(\mathbb{E}\left[X_{k}^{2}+2 X_{k}+1\right]\right)^{1 / 2} \leq s^{2} L_{0}^{2} C .
\end{aligned}
$$

The above result is based on the Cauchy-Schwarz inequality and norm subadditivity. Moreover, the last inequality uses 3.1 which also proves that the expectations of powers of logarithms of diagonal Green functions are uniformely bounded by Lemma 3.6 . 
In applying Lemma 3.3 it is also useful to note that

$$
\begin{aligned}
& \mathbb{E}\left[\ln \prod_{k=0}^{N_{x}-1} X_{k}\right]-s \mathbb{E}\left(\ln \left|\left\langle\delta_{0},\left(H_{\mathcal{T}_{L}}-E\right)^{-1} \delta_{x}\right\rangle\right|\right) \\
& =-\sum_{k=0}^{N_{x}-1} \mathbb{E}\left[\ln Y_{k}\right]-\mathbb{E}[\ln R] \\
& \leq N_{x} \max _{k}\left|\mathbb{E}\left[\ln Y_{k}\right]\right|+s L_{0} \max _{j}\left|\mathbb{E}\left[\ln \left|\Gamma_{j, L}\right|\right]\right| \leq s C\left(N_{x}+L_{0}\right),
\end{aligned}
$$

where we have again used the fact that expectations of logarithms of diagonal Green functions are uniformely bounded.

Summarizing the above estimates we obtain the bound

$$
\begin{aligned}
& \ln \mathbb{E}\left[\left|\left\langle\delta_{0},\left(H_{\mathcal{T}_{L}}-E\right)^{-1} \delta_{x}\right\rangle\right|^{s}\right]-s \mathbb{E}\left(\ln \left|\left\langle\delta_{0},\left(H_{\mathcal{T}_{L}}-E\right)^{-1} \delta_{x}\right\rangle\right|\right) \\
& \leq\left(N_{x}+1\right) \ln C_{s}+N_{x} s^{2} L_{0}^{2} C+s C\left(N_{x}+L_{0}\right) \\
& \leq \operatorname{dist}(0, x)\left(2 L_{0}^{-1} \ln C_{s}+s^{2} L_{0} C+2 s C\right),
\end{aligned}
$$

where the last inequality holds provided $\operatorname{dist}(0, x) \geq L_{0}$. Consequently, for a given $\varepsilon>0$ we may then pick $L_{0}=L_{\varepsilon}$ large enough and $s_{\varepsilon}$ small enough such that the right side in (3.14) is smaller that $\varepsilon \operatorname{dist}(0, x)$ for every $s \in\left(0, s_{\varepsilon}\right)$.

We close this subsection by compiling two elementary estimates on expectations of functions of the diagonal of the Green function. The first bounds concern fractional moments of the Green function going back to [AM93].

Lemma 3.5. Assume $\mathbf{A} 1$ and let $s \in(0,1), z \in \mathbb{C}$ and $L \in \mathbb{N}$. Then

$$
\mathbb{E}\left[\left|\left\langle\delta_{0},\left(H_{\mathcal{T}_{L}}-z\right)^{-1} \delta_{0}\right\rangle\right|^{s}\right] \leq C_{s}
$$

and

$$
\mathbb{E}\left[\left|\left\langle\delta_{0},\left(H_{\mathcal{T}_{L}}-z\right)^{-1} \delta_{0}\right\rangle\right|^{-s}\right] \leq \int_{\mathbb{R}}|\xi|^{s} \varrho(\xi) d \xi+|z|^{s}+K C_{s},
$$

where $C_{s}$ is the constant appearing in 3.1..

Proof. The first inequality is an immediate consequence of the fractional moment bound (3.1). The second one is a consequence of the first and the recursion relation which the diagonal of the resolvent is well-known satisfy, cf. [K198],

$$
\left\langle\delta_{0},\left(H_{\mathcal{T}_{L}}-z\right)^{-1} \delta_{0}\right\rangle=\left(\omega_{0}-z-\sum_{\operatorname{dist}(x, 0)=1}\left\langle\delta_{x},\left(H_{\mathcal{T}_{L}(x)}-z\right)^{-1} \delta_{x}\right\rangle\right)^{-1}
$$

where we recall that $\mathcal{T}_{L}(x)$ is that subtree of $\mathcal{T}_{L}$ which is forward to $x$.

Lemma 3.5 in particular implies that any moment of the logarithm of the Green function is uniformly bounded. 
Lemma 3.6. Assume $\mathbf{A} 1$ and let $I \subset \mathbb{R}$ be a bounded Borel set and $n \in \mathbb{Z}$. Then

$$
\sup _{E \in I} \sup _{L \in \mathbb{N}} \mathbb{E}\left[|\ln |\left\langle\delta_{0},\left(H_{\mathcal{T}_{L}}-E\right)^{-1} \delta_{0}\right\rangle||^{n}\right]<\infty .
$$

Proof. This estimate immediately follows from Lemma3.5 and the fact that $|\ln \xi| \leq$ $\xi^{\tau}+\xi^{-\tau}$ for any $\xi>0$ and $\tau \neq 0$.

\subsection{Lower bound on the Lyapunov exponent}

In [ASW06a] we considered a Lyapunov exponent for the operator $H_{\mathcal{T}}$ on the infinite regular rooted tree with branching number $K \geq 2$, defining it as

$$
\gamma(z):=-\mathbb{E}\left[\ln \left(\sqrt{K} \mid\left\langle\delta_{0},\left(H_{\mathcal{T}}-z\right)^{-1} \delta_{0}\right\rangle\right) \mid\right] .
$$

It was shown there ([ASW06a, Thm. $3.1 \&$ Thm. 4.1]) that

1. $\gamma(z)$ is a positive harmonic function of $z \in \mathbb{C}^{+}$and hence its boundary values $\gamma(E+i 0)$ with $E \in \mathbb{R}$ define a locally integrable function.

2. For all $z \in \mathbb{C}^{+}$and all $\alpha \in(0,1 / 2)$

$$
\gamma(z) \geq \frac{\alpha^{2}}{32(K+1)^{2}}\left(\delta\left(\left|\Gamma_{0}(z)\right|^{-2}, \alpha\right)\right) 2,
$$

where $\Gamma_{0}(z):=\left\langle\delta_{0},\left(H_{\mathcal{T}}-z\right)^{-1} \delta_{0}\right\rangle$ and $\delta(\cdot, \cdot)$ is defined as follows.

Definition 3.1 (Relative width). For $\alpha \in(0,1 / 2]$ the relative $\alpha$-width of a positive random variable $X$ is given by

$$
\delta(X, \alpha):=1-\frac{\xi_{-}(X, \alpha)}{\xi_{+}(X, \alpha)} .
$$

where $\xi_{-}(X, \alpha):=\sup \{\xi, \mathbb{P}(X<\xi) \leq \alpha\}$ and $\xi_{+}(X, \alpha):=\inf \{\xi, \mathbb{P}(X>\xi) \leq$ $\alpha\}$.

Our next task is to further estimate the right side of (3.20) from below. This will be done with the help of the following lemma.

Lemma 3.7. Let $X$ be a positive random variable with probability measure $\mathbb{P}$. Suppose

1. there exists $\sigma \in(0,1]$ and $C_{\sigma}<\infty$ such that $\mathbb{P}(X \in I) \leq C_{\sigma}|I|^{\sigma}$ for all Borel sets $I \subset[0, \infty)$ with $|I| \leq 1$.

2. there exists $\tau>0$ such that $\mathbb{E}\left[X^{\tau}\right]<\infty$.

Then for all $\alpha \in(0,1 / 2)$

$$
\delta(X, \alpha) \geq \min \left\{1,\left(\frac{1-2 \alpha}{C_{\sigma}}\right)^{1 / \sigma}\right\}\left(\frac{\alpha}{\mathbb{E}\left[X^{\tau}\right]}\right)^{1 / \tau} .
$$


Proof. The first assumption implies that $1-2 \alpha=\mathbb{P}\left\{X \in\left(\xi_{-}(X, \alpha), \xi_{+}(X, \alpha)\right)\right\} \leq$ $C_{\sigma}\left(\xi_{+}(X, \alpha)-\xi_{-}(X, \alpha)\right)^{\sigma}$ provided $\xi_{+}(X, \alpha)-\xi_{-}(X, \alpha) \leq 1$. From the second assumption we conclude that $\alpha \leq \mathbb{P}\left\{X \in\left(\xi_{+}(X, \alpha), \infty\right)\right\} \leq \mathbb{E}\left[X^{\tau}\right] / \xi_{+}(X, \alpha)^{\tau}$ by a Chebychev inequality. Inserting these two estimates into 3.21 completes the proof.

Lemma 3.8. Let $I \subset \mathbb{R}$ be a bounded Borel set and

$$
l(I):=\sup _{\alpha \in(0,1 / 2)} \frac{\alpha^{2}}{32(K+1)^{2}} \min \left\{1, \frac{1-2 \alpha}{2\|\varrho\|_{\infty}}\right\}\left(\frac{\alpha}{\mathbb{E}\left[\sup _{E \in I}\left|\Gamma_{0}(E+i 0)\right|^{-\tau}\right]}\right)^{2 / \tau}
$$

where $\tau$ is the constant appearing in Assumption A1. Then $\gamma(E+i 0) \geq l(I)>0$ for any $E \in I$.

Proof. In order to apply Lemma 3.7 to the right side in 3.20 we need to check its assumptions. We first note that by the Krein formula $\left|\Gamma_{0}(z)\right|^{-2}=\left(\omega_{0}-a\right)^{2}+b^{2}$ with suitable $a, b \in \mathbb{R}$. An elementary computation shows that for every Borel set $I \subset[0, \infty)$ with $|I| \leq 1$

$$
\int_{\mathbb{R}} \varrho(\xi) 1_{\left\{(\xi-a)^{2}+b^{2} \in I\right\}} d \xi \leq \int_{I} \frac{\|\varrho\|_{\infty}}{\sqrt{\xi-b 2}} 1_{\left\{\xi \geq b^{2}\right\}} d \xi \leq 2\|\varrho\|_{\infty} \sqrt{|I|} .
$$

Moreover, Lemma 3.5 guarantees that $\sup _{E \in I} \mathbb{E}\left[\left|\Gamma_{0}(E+i 0)\right|^{-\tau}\right]<\infty$.

Associated with $\gamma(z)$ is the following finite-volume approximation

$$
\gamma_{L}(z):=-\mathbb{E}\left[\ln \left(\sqrt{K} \mid\left\langle\delta_{0},\left(H_{\mathcal{T}_{L}}-z\right)^{-1} \delta_{0}\right|\right)\right] .
$$

It is easy to see that $\gamma_{L}(z)$ also defines a harmonic function of $z \in \mathbb{C}^{+}$. Moreover, its boundary values $\gamma_{L}(E)$ are defined everywhere by setting $z=E \in \mathbb{R}$ in (3.25). Strong resolvent convergence implies that $\lim _{L \rightarrow \infty} \gamma_{L}(z)=\gamma(z)$ for every $z \in \mathbb{C}^{+}$. Assumption $\mathbf{A 2}$ guarantees that this convergence holds and is locally uniform also for real arguments.

Lemma 3.9. Suppose $\mathbf{A} 2$ holds for a bounded Borel set $I \subset \mathbb{R}$. Then

$$
\lim _{L \rightarrow \infty} \sup _{E \in I}\left|\gamma_{L}(E)-\gamma(E+i 0)\right|=0 .
$$

Proof. Since $\gamma_{L}(E)$ are uniformly bounded for $E \in I$, cf. Lemma3.6. By the ArzelaAscoli theorem Assumption A2 thus implies that every subsequence of $\gamma_{L}$ has a uniformly convergent subsequence. The claim (3.26) then follows by showing that any pointwise limit of $\gamma_{L}(E)$ coincides with $\gamma(E+i 0)$. This is derived from the above mentioned strong resolvent convergence and the dominated convergence theorem, which imply that for any bounded and compactly supported function $\phi \in L_{c}^{\infty}(\mathbb{R})$

$$
\int_{\mathbb{R}} \gamma(E+i 0) \phi(E) d E=\lim _{L \rightarrow \infty} \int_{\mathbb{R}} \gamma_{L}(E) \phi(E) d E=\int_{\mathbb{R}} \lim _{L \rightarrow \infty} \gamma_{L}(E) \phi(E) d E .
$$

provided $\lim _{L \rightarrow \infty} \gamma_{L}(E)$ exists for Lebesgue-almost all $E \in \mathbb{R}$. 


\subsection{Proof of Theorem 3.2}

Proof of Theorem 3.2 If $x \neq y$ is in the future of $y$, the Green function factorizes according to

$$
\left\langle\delta_{y},\left(H_{\mathcal{T}_{L}}-E\right)^{-1} \delta_{x}\right\rangle=\left\langle\delta_{y},\left(H_{\mathcal{T}_{L}}-E\right)^{-1} \delta_{y}\right\rangle\left\langle\delta_{v},\left(H_{\mathcal{T}_{L}(v)}-E\right)^{-1} \delta_{x}\right\rangle
$$

where $v$ is that forward neighbor of $y$ which lies on the unique path connecting $x$ and $y$. We may therefore suppose without loss of generality that $y$ coincides with the root in $\mathcal{T}_{L}$.

In this case, Lemma 3.4 bounds the fractional moment of the Green function by an exponential involving

$$
\mathbb{E}\left[\ln \left\langle\delta_{y},\left(H_{\mathcal{T}_{L}}-E\right)^{-1} \delta_{x}\right\rangle\right]=-\sum_{j=0}^{\operatorname{dist}(y, x)}\left(\gamma_{L-j}(E)+\ln \sqrt{K}\right),
$$

where we the last equality results from 3.5 , stationarity and the definition of the finitevolume Lyapunov exponent in 3.25). According to Lemma3.9 for a given $\varepsilon>0$ there exists $L_{\varepsilon} \in \mathbb{N}$ such that $\gamma_{L}(E) \geq \gamma(E+i 0)-\varepsilon \geq l(I)-\varepsilon$ for all $E \in I$ and $L \geq L_{\varepsilon}$, where $l(I)>0$ was defined in Lemma 3.8 The proof is completed by choosing $\varepsilon$ small enough in the last estimate and in Lemma 3.4

\section{Proof of Poisson statistics for tree operators}

We will follow the general strategy outlined in Subsection 2.2. Poisson statistics are established through the proof of the negligibility in probability condition 2.5 , which holds due to the divergence of fluctuations of the value at a fixed site of the normalized eigenfunctions.

\subsection{Divergent fluctuations of the spectral measure in the bulk}

The following theorem allows to conclude that for any $x \in \mathcal{T}$ the sequence of spectral measures $\left\{\sigma_{x, L}\right\}$ has divergent fluctuations in the sense of Definition 2.1, at any $E \in \mathbb{R}$ for which the average over the disorder of theses measures is non zero.

Theorem 4.1 (Negligibility in probability). Assume $\mathbf{A 1}$ and $\mathbf{A 2}$ holds for a bounded Borel set $I \subset \mathbb{R}$. If $I_{L} \subset I$ are bounded Borel sets such that $\lim _{\sup } \sup _{L \rightarrow \infty}\left|I_{L}\right|\left|\mathcal{T}_{L}\right|<$ $\infty$, then for any $x \in \mathcal{T}$

$$
\mathbb{P}-\lim _{L \rightarrow \infty}\left|\mathcal{T}_{L}\right| \sigma_{x, L}\left(I_{L}\right)=0 .
$$

For the proof we now fix $x \in \mathcal{T}$, and for $L \in \mathbb{N}$ large enough so that $x \in \mathcal{T}_{L}$ and every $y \in \mathcal{T}_{L}$ we define the ratio

$$
g_{y, L}(E):=\frac{\left|\left\langle\delta_{x},\left(H_{\mathcal{T}_{L}}-E\right)^{-1} \delta_{y}\right\rangle\right|^{2}}{\left\langle\delta_{y},\left(H_{\mathcal{T}_{L}}-E\right)^{-2} \delta_{y}\right\rangle}
$$


It is well-defined for Lebesgue-almost all $E \in \mathbb{R}$. Moreover, by the rank-one pertubation formula and the spectral theorem it is seen to enjoys the following properties:

1. $g_{y, L}(E)$ is independent of the value of the potential at $y \in \mathcal{T}_{L}$.

2. The function $E \mapsto g_{y, L}(E)$ has a continuous extension on $\mathbb{R}$. Moreover, if the eigenvalue $E_{n}\left(\mathcal{T}_{L}\right)$ of $H_{\mathcal{T}_{L}}$ is non-degenerate, then the corresponding eigenfunction satisfies

$$
\left|\left\langle\delta_{x}, \psi_{n}\left(\mathcal{T}_{L}\right)\right\rangle\right|^{2}=\lim _{E \rightarrow E_{n}\left(\mathcal{T}_{L}\right)} g_{y, L}(E)
$$

Theorem 4.1 will now be a consequence of the following result.

Lemma 4.2. Under the assumptions of Theorem 4.1 for any $\varepsilon>0$

$$
\lim _{L \rightarrow \infty} \sum_{y \in \mathcal{T}_{L}} \mathbb{E}\left[\sigma_{y, L}\left(E \in I_{L}: g_{y, L}(E) \geq \varepsilon\left|\mathcal{T}_{L}\right|^{-1}\right)\right]=0 .
$$

Proof. The proof is based on the spectral averaging principle (cf. [SW86, CL90]),

$$
\int_{\mathbb{R}} \varrho\left(\omega_{y}\right) \sigma_{y, L}(I) d \omega_{y} \leq\|\varrho\|_{\infty} \int_{\mathbb{R}} \sigma_{y, L}(I) d \omega_{y} \leq\|\varrho\|_{\infty}|I|
$$

for all bounded Borel sets $I \subset \mathbb{R}$. Using this inequality and the fact that $g_{y, L}(E)$ does not depend on $\omega_{y}$, the prelimit in (4.4) can be bounded from above by

$$
\|\varrho\|_{\infty} \sum_{y \in \mathcal{T}_{L}} \int_{I_{L}} \mathbb{P}\left(g_{y, L}(E) \geq \varepsilon\left|\mathcal{T}_{L}\right|^{-1}\right) d E .
$$

We now pick $N \in \mathbb{N}$ and split the summation in (4.6) into two terms. The first term collects all contributions corresponding to $\mathcal{T}_{L-N} \subset \mathcal{T}_{L}$,

$$
\sum_{y \in \mathcal{T}_{L-N}} \int_{I_{L}} \mathbb{P}\left(g_{y, L}(E) \geq \varepsilon\left|\mathcal{T}_{L}\right|^{-1}\right) d E \leq\left|\mathcal{T}_{L-N}\right|\left|I_{L}\right|
$$

In the limit $L \rightarrow \infty$ this term is arbitrarily small for $N$ large enough. To estimate the remaining second term, we abbreviate $\alpha_{y, L}(E):=\left\langle\delta_{y},\left(H_{\mathcal{T}_{L}}-E\right)^{-2} \delta_{y}\right\rangle$ and write

$$
\begin{aligned}
\mathbb{P}\left(g_{y, L}(E)\right. & \left.\geq \varepsilon\left|\mathcal{T}_{L}\right|^{-1}\right)=\mathbb{P}\left(\left|\mathcal{T}_{L}\right|\left|\left\langle\delta_{x},\left(H_{\mathcal{T}_{L}}-E\right)^{-1} \delta_{y}\right\rangle\right|^{2} \geq \varepsilon \alpha_{y, L}(E)\right) \\
& \leq \mathbb{P}\left(\left|\mathcal{T}_{L}\right|\left|\left\langle\delta_{x},\left(H_{\mathcal{T}_{L}}-E\right)^{-1} \delta_{y}\right\rangle\right|^{2} \geq \varepsilon \alpha\right)+\mathbb{P}\left(\alpha_{y, L}(E)<\alpha\right)
\end{aligned}
$$

where the last inequality holds for any $\alpha \in(0, \infty)$. The first term on the right side of (4.8) gives rise to the following contribution to the sum in (4.6),

$$
\begin{aligned}
\sum_{y \in \mathcal{T}_{L} \backslash \mathcal{T}_{L-N}} & \int_{I_{L}} \mathbb{P}\left(\left|\mathcal{T}_{L}\right|\left|\left\langle\delta_{x},\left(H_{\mathcal{T}_{L}}-E\right)^{-1} \delta_{y}\right\rangle\right|^{2} \geq \varepsilon \alpha\right) d E \leq \frac{\left|\mathcal{T}_{L}\right|^{s}}{\varepsilon^{s} \alpha^{s} K^{s(L-N)}} \\
& \times \sup _{E \in I}\left|I_{L}\right| \sum_{y \in \mathcal{T}_{L} \backslash \mathcal{T}_{L-N}} K^{s \operatorname{dist}(x, y)} \mathbb{E}\left[\left|\left\langle\delta_{x},\left(H_{\mathcal{T}_{L}}-E\right)^{-1} \delta_{y}\right\rangle\right|^{2 s}\right]
\end{aligned}
$$


where $I \subset \mathbb{R}$ is some bounded Borel set which contains eventually all $I_{L}$. While the prefactors on the right side of 4.9) remains finite in the limit $L \rightarrow \infty$, the supremum converges to zero in this limit, since it is bounded by $\left|I_{L}\right|\left|\mathcal{T}_{L}\right| C \exp (-2 s \delta(L-N))$ for sufficently small $s$ by Theorem 3.2 To complete we note that the second term in (4.8), converges to zero as $\alpha \downarrow 0$, uniformly in $E \in I, L \in \mathbb{N}$ and $y \in \mathcal{T}_{L}$. This follows from the bound

$$
\begin{aligned}
\mathbb{P}\left(\alpha_{y, L}(E)<\alpha\right) & \leq \mathbb{P}\left(\left\|\left(H_{\mathcal{T}_{L}}-E\right) \delta_{y}\right\|^{-2}<\alpha\right) \\
& \leq \alpha^{s}\left(\left\|(A+B-E) \delta_{y}\right\|^{2 s}+\mathbb{E}\left[\left|\omega_{y}\right|^{2 s}\right]\right),
\end{aligned}
$$

where the last step requires $2 s<\min (1, \tau)$.

Proof of Theorem 4.1 Wegner's bound 2.1 implies that $I_{L}$ carries only a finite number of eigenvalues

$$
\lim _{N \rightarrow \infty} \sup _{L \in \mathbb{N}} \mathbb{P}\left(\operatorname{Tr} P_{I_{L}}\left(H_{\mathcal{T}_{L}}\right) \geq N\right)=0 .
$$

It therefore remains to prove that for any $\varepsilon>0$

$$
\lim _{L \rightarrow 0} \mathbb{E}\left[\sum_{E_{n}\left(\mathcal{T}_{L}\right) \in I_{L}} 1\left\{\left|\left\langle\delta_{x}, \psi_{n}\left(\mathcal{T}_{L}\right)\right\rangle\right|^{2} \geq \varepsilon\left|\mathcal{T}_{L}\right|^{-1}\right\}\right]=0,
$$

where $1\{\cdots\}$ stands for the indicator function. Using the fact that $H_{\mathcal{T}_{L}}$ has almost surely no degenerate eigenfunctions (cf. Proposition 2.1) and (4.3), the left side in (4.12) is seen to be equal to the left side in (4.4).

Theorem 2.2 now guarantees that any accumulation point of $\left\{\mu_{L}^{E}\right\}$ is a random Poisson measure. The uniqueness of the accumulation point will be proven by establishing uniqueness of the intensity measure, which is defined next.

\subsection{The intensity measure}

For a random point measure $\mu$ the intensity measure is defined as the average

$$
\bar{\mu}:=\mathbb{E}[\mu] .
$$

Thus $\bar{\mu}_{L}^{E}$ are the intensity measures of the random point measures $\mu_{L}^{E}$. We shall also use the symbol $\bar{\mu}^{E}$ for the intensity measure of a given accumulation point of the sequence $\left\{\mu_{L}^{E}\right\}$.

Let us proceed with a more explicit representation for $\bar{\mu}_{L}^{E}$. For any Borel set $I \subset \mathbb{R}$ we have

$$
\begin{aligned}
\bar{\mu}_{L}^{E}(I) & =\mathbb{E}\left[\operatorname{Tr} P_{E+I /\left|\mathcal{T}_{L}\right|}\left(H_{\mathcal{T}_{L}}\right)\right] \\
& =\sum_{x \in \mathcal{T}_{L}} \mathbb{E}\left[\left\langle\delta_{x}, P_{E+I /\left|\mathcal{T}_{L}\right|}\left(H_{\mathcal{T}_{L}}\right) \delta_{x}\right\rangle\right] \\
& =\sum_{n=0}^{L} K^{L-n} \mathbb{E}\left[\left\langle\delta_{x_{n}}, P_{E+I\left|\mathcal{T}_{L}\right|^{-1}}\left(H_{\mathcal{T}_{L}}\right) \delta_{x_{n}}\right\rangle\right],
\end{aligned}
$$


where we used the fact that the expectation in the second line does not depend on $x$ as long as $\operatorname{dist}(0, x)$ is constant. Moreover, $x_{n}$ denotes any vertex with $\operatorname{dist}\left(x_{n}, \partial \mathcal{T}_{L}\right)=$ $n$. In view of LemmaA.1 in the appendix, the above calculation (4.14) suggests that the intensity measure $\bar{\mu}_{L}^{E}$ converges for Lebesgue almost all $E \in \mathbb{R}$ to Lebesgue measure times the canopy density of states given by

$$
d_{\mathcal{C}}(E):=\frac{K-1}{K} \sum_{n=0}^{\infty} K^{-n} \pi^{-1} \mathbb{E}\left[\operatorname{Im}\left\langle\delta_{x_{n}},\left(H_{\mathcal{C}}-E-i 0\right)^{-1} \delta_{x_{n}}\right\rangle\right] .
$$

Theorem 4.3 (Limiting intensity measure). Under assumption $\mathbf{A 1}$ and $\mathbf{A 2}$ (or alternatively A1' below) for Lebesgue-almost all $E \in \mathbb{R}$ the intensity measure $\bar{\mu}^{E}$ of any weak accumulation point $\mu^{E}$ of the sequence $\mu_{L}^{E}$ is given by

$$
\bar{\mu}^{E}(I)=\lim _{L \rightarrow \infty} \bar{\mu}_{L}^{E}(I)=d_{\mathcal{C}}(E)|I|
$$

for all bounded Borel sets $I \subset \mathbb{R}$.

Proof. As an immediate consequence of Wegner's estimate 2.1. and the first line in (4.14) we have that for Lebesgue-almost all $E \in \mathbb{R}$ and all $L \in \mathbb{N}$ the measures $\bar{\mu}_{L}^{E}$ are absolutely continuous with bounded density,

$$
\frac{\bar{\mu}_{L}^{E}(d \xi)}{d \xi} \leq\|\varrho\|_{\infty} .
$$

The same applies to any accumulation point $\bar{\mu}^{E}$. As a consequence, the linear functional given by $\bar{\mu}_{L}^{E}(\psi):=\int_{\mathbb{R}} \psi(\xi) \bar{\mu}_{L}^{E}(d \xi)$ is uniformly equicontinuous on the space of non-negative integrable functions on the real line, $\psi \in L_{+}^{1}(\mathbb{R})$. More precisely, (4.17) yields

$$
\left|\bar{\mu}_{L}^{E}(\phi)-\bar{\mu}_{L}^{E}(\psi)\right| \leq\|\varrho\|_{\infty}\|\phi-\psi\|_{1}
$$

for all $\phi, \psi \in L_{+}^{1}(\mathbb{R})$. Using this and the fact that the functions $\varphi_{z}:=\pi^{-1} \operatorname{Im}(\cdot-z)^{-1}$ with $z \in \mathbb{C}^{+}$are dense in $L_{+}^{1}(\mathbb{R})$ implies that it suffices to check $(4.16)$ if the indicator function of $I$ is replaced by $\varphi_{z}$.

Moreover, elementary considerations show that it suffices to verify

$$
\lim _{L \rightarrow \infty} \int_{\mathbb{R}}\left|\bar{\mu}_{L}^{E}\left(\varphi_{z}\right)-d(E)\left\|\varphi_{z}\right\|_{1}\right| d E=0
$$

with $z \in \mathbb{C}^{+}$fixed but arbitrary. A computation similiar to (4.14) and the fact that $\left\|\varphi_{z}\right\|_{1}=1$ then proves that this derives from

$$
\begin{aligned}
\lim _{L \rightarrow \infty} \int_{\mathbb{R}} \mathbb{E}\left[\mid \operatorname{Im}\left\langle\delta_{x},\left(H_{\mathcal{T}_{L}}-E-z\left|\mathcal{T}_{L}\right|^{-1}\right)^{-1} \delta_{x}\right\rangle\right. & \\
& \left.-\operatorname{Im}\left\langle\delta_{x},\left(H_{\mathcal{C}}-E-i 0\right)^{-1} \delta_{x}\right\rangle \mid\right] d E=0
\end{aligned}
$$

for $x \in \mathcal{C}$ with $\operatorname{dist}(x, \partial \mathcal{C}) \in \mathbb{N}_{0}$ fixed but arbitrary.

For a proof of (4.20), we appeal to Riesz's theorem which guarantees that the claimed $L^{1}$-convergence follows from the almost sure convergence of the integrand 
in (4.20) with respect to the product of the probability measure and Lebesgue measure, and the equality of the integrals

$$
\begin{aligned}
\lim _{L \rightarrow \infty} \frac{1}{\pi} \int_{\mathbb{R}} \mathbb{E}[\operatorname{Im} & \left.\left\langle\delta_{x},\left(H_{\mathcal{T}_{L}}-E-z\left|\mathcal{T}_{L}\right|^{-1}\right)^{-1} \delta_{x}\right\rangle\right] d E \\
& =\frac{1}{\pi} \int_{\mathbb{R}} \mathbb{E}\left[\operatorname{Im}\left\langle\delta_{x_{n}},\left(H_{\mathcal{C}}-E-i 0\right)^{-1} \delta_{x_{n}}\right\rangle\right] d E=1 .
\end{aligned}
$$

In fact, we only need to show that the integrand in 4.20 converges in distribution with respect to the product measure. To prove the latter we first note that one has the non-tangential limit

$$
\lim _{L \rightarrow \infty}\left\langle\delta_{x},\left(H_{\mathcal{C}}-E-z\left|\mathcal{T}_{L}\right|^{-1}\right)^{-1} \delta_{x}\right\rangle=\left\langle\delta_{x},\left(H_{\mathcal{C}}-E-i 0\right)^{-1} \delta_{x}\right\rangle
$$

for Lebesgue-almost all $E \in \mathbb{R}$. Moreover, using the resolvent identity twice, we obtain the inequality

$$
\begin{array}{r}
\left|\left\langle\delta_{x},\left(H_{\mathcal{T}_{L}}-E-z\left|\mathcal{T}_{L}\right|^{-1}\right)^{-1} \delta_{x}\right\rangle-\left\langle\delta_{x},\left(H_{\mathcal{C}}-E-z\left|\mathcal{T}_{L}\right|^{-1}\right)^{-1} \delta_{x}\right\rangle\right| \\
\leq\left|\left\langle\delta_{x},\left(H_{\mathcal{T}_{L}}-E-z\left|\mathcal{T}_{L}\right|^{-1}\right)^{-1} \delta_{0_{L}}\right\rangle\left\langle\delta_{0_{L}},\left(H_{\mathcal{T}_{L}}-E-z\left|\mathcal{T}_{L}\right|^{-1}\right)^{-1} \delta_{x}\right\rangle\right| \\
\times\left|\left\langle\delta_{0_{L}^{-}},\left(H_{\mathcal{C}}-E-z\left|\mathcal{T}_{L}\right|^{-1}\right)^{-1} \delta_{0_{L}^{-}}\right\rangle\right|
\end{array}
$$

where $0_{L}$ is the root in $\mathcal{T}_{L}$ and $0_{L}^{-}$is its backward neighbor. The right side converges to zero in distribution with respect to the product of the probability measure and Lebesgue measure on any bounded interval. This follows from Theorem 3.2 (or alternatively Proposition 6.2 below) and the fact that the factional-moment bound (3.1) implies that the probability that the last term in (4.23) is large is bounded.

\subsection{Proof of Theorem 1.1}

Theorem 1.1 may be stated using the characterisation of the Poisson process in terms of its characteristic functional. Namely, the random measure $\mu^{E}$ is Poisson if for any bounded Borel set $I \subset \mathbb{R}$ and $t \geq 0$

$$
\mathbb{E}\left[e^{-t \mu^{E}(I)}\right]=\exp \left(-\mathbb{E}\left[\mu^{E}(I)\right]\left(1-e^{-t}\right)\right) .
$$

Given Theorem 2.2 and Theorem 4.1 the proof of 4.24 is basically a repetition of well-known arguments how to conclude the Poisson nature of accumulation points from infinite divisibility and the exclusion of double points [ $\mathrm{Ka02}]$.

Proof of Theorem 1.1] Let $\mu^{E}$ be an accumulation point of $\left\{\mu_{L}^{E}\right\}$. Theorem 2.2 implies that for any $N \in \mathbb{N}$ and any bounded Borel set $I \subset \mathbb{R}$

$$
\begin{aligned}
\lim _{L \rightarrow \infty} \mathbb{E}\left[\exp \left(-t \sum_{\operatorname{dist}(0, x)=N} \mu_{x, L}^{E}(I)\right)\right] \\
=\lim _{L \rightarrow \infty} \mathbb{E}\left[e^{-t \mu_{L}^{E}(I)}\right]=\mathbb{E}\left[e^{-t \mu^{E}(I)}\right] .
\end{aligned}
$$


Since the measures in the left side of 4.25) are iid, the expectation factorizes into a $K^{N}$-fold product of

$$
\begin{aligned}
\mathbb{E}\left[e^{-t \mu_{x, L}^{N}(I)}\right] & =\sum_{m=0}^{\infty} e^{-t m} \mathbb{P}\left(\mu_{x, L}^{E}(I)=m\right) \\
& =1-\mathbb{E}\left[\mu_{x, L}^{E}(I)\right]\left(1-e^{-t}\right)+R_{x, L}(I),
\end{aligned}
$$

where

$$
\begin{aligned}
0 \leq R_{x, L}(I) & :=\sum_{m=2}^{\infty} \mathbb{P}\left(\mu_{x, L}^{E}(I)=m\right)\left[m\left(1-e^{-t}\right)+e^{-t m}-1\right] \\
& \leq \sum_{m=2}^{\infty}(m-1) \mathbb{P}\left(\mu_{x, L}^{E}(I)=m\right)=\sum_{m=2}^{\infty} \mathbb{P}\left(\mu_{x, L}^{E}(I) \geq m\right)
\end{aligned}
$$

By (2.2) this term is arbitrarily small in the limit $L \rightarrow \infty$ provided $N$ is large enough. The second term in (4.26) converges,

$$
\lim _{L \rightarrow \infty} K^{N} \mathbb{E}\left[\mu_{x, L}^{E}(I)\right]=\mathbb{E}\left[\mu^{E}(I)\right] .
$$

The claim now follows by taking the subsequent limit $N \rightarrow \infty$ in 4.25 from the fact that $\lim _{n \rightarrow \infty}\left(1+x_{n} / n\right)^{n}=e^{x}$ for any complex-valued sequence with $\lim _{n \rightarrow \infty} x_{n}=$ $x$.

\section{Complete localization for random operators on the canopy graph}

We shall now prove Theorem 1.3, which asserts that, under assumptions $\mathbf{A 1}$ and $\mathbf{A 2}$, on the canopy graph the random Schrödinger operator has only pure point spectrum, i.e., a complete set of square integrable eigenfunctions. The argument is based on the Simon-Wolff criterion [SW86], for which a sufficient condition is that for every energy the Green function be almost surely square summable, when summed over one of its arguments. (Through spectral averaging a.s. properties of the Green function areshared by the eigenfunctions.)

Applying the above criterion, an intuitive reason for localization on the canopy graph is that the number of points at distance $n$ from $x_{0}$ grows there as $K^{n / 2}$, which is square root of the corresponding number for the regular tree. By Theorem 3.2 , the Green function decays at a rate which - if one could ignore large deviations, would yield square summability. That in itself is not enough since typically the sum of the Green function is much larger that the sum of the typical values - otherwise, the result would be valid also for the full homogeneous tree. While the corresponding statement is not valid in that case, square summability is missed there rather marginally. Thus it may be not that surprising that the significant reduction in the number of sites suffices to yield the required summability. We establish that with the help of a fractional moment estimate, and making use of the two lemmas which follow. 
We now regard $\mathcal{T}_{L}$ as being embedded into $\mathcal{C}$ in such a way that the outer boundary $\partial \mathcal{T}_{L}$ is embedded into $\partial \mathcal{C}$ for every $L \in \mathbb{N}$.

Lemma 5.1. Assume $\mathbf{A 1}$ and $\mathbf{A 2}$ holds for a bounded Borel set $I \subset \mathbb{R}$. Then there exists $s \in(0,1)$ such that for all $x \in \mathcal{C}$ and Lebesgue-almost all $E \in I$

$$
\sup _{\eta \neq 0} \sup _{L \geq L_{x}} \mathbb{E}\left[\left\langle\delta_{x},\left|H_{\mathcal{T}_{L}}-E-i \eta\right|^{-2} \delta_{x}\right\rangle^{s}\right]<\infty,
$$

where $L_{x}:=\min \left\{L \in \mathbb{N}: x \in \mathcal{T}_{L}\right\}$.

Proof. We first note that the inequality

$$
\left\langle\delta_{x},\left|H_{\mathcal{T}_{L}}-E-i \eta\right|^{-2} \delta_{x}\right\rangle \leq\left\langle\delta_{x},\left|H_{\mathcal{T}_{L}}-E\right|^{-2} \delta_{x}\right\rangle
$$

implies that we only need to bound the $\ell^{2}\left(\mathcal{T}_{L}\right)$-norm in 5.1) for $\eta=0$. The expectation of the fractional-moment of this $\ell^{2}\left(\mathcal{T}_{L}\right)$-norm is split into two contributions. One involves all terms corresponding to the finite subtree

$$
\mathcal{C}(x):=\{y \in \mathcal{C}: y \text { is forward (in the direction of } \partial \mathcal{C} \text { ) or equal to } x\},
$$

which has $x$ as its root, and the other collects all remaining terms. Employing the elementary inequality $\left(\sum_{j} \alpha_{j}\right)^{s} \leq \sum_{j} \alpha_{j}^{s}$, which is valid for any $s \in(0,1)$ and any collection of non-negative numbers $\alpha_{j}$, we thus obtain

$$
\begin{aligned}
\mathbb{E}\left[\left(\sum_{y \in \mathcal{T}_{L}}\left|\left\langle\delta_{x},\left(H_{\mathcal{T}_{L}}-E\right)^{-1} \delta_{y}\right\rangle\right|^{2}\right)^{s}\right] \leq S_{1}+S_{2}, \\
\text { where } S_{1}:=\sum_{y \in \mathcal{C}(x)} \mathbb{E}\left[\left|\left\langle\delta_{x},\left(H_{\mathcal{T}_{L}}-E\right)^{-1} \delta_{y}\right\rangle\right|^{2 s}\right] \\
S_{2}:=\mathbb{E}\left[\left(\sum_{y \in \mathcal{T}_{L} \backslash \mathcal{C}(x)}\left|\left\langle\delta_{x},\left(H_{\mathcal{T}_{L}}-E\right)^{-1} \delta_{y}\right\rangle\right|^{2}\right)^{s}\right] .
\end{aligned}
$$

By the fractional-moment bound (3.1) the first terms, $S_{1}$, is bounded for any $s \in$ $(0,1 / 2)$ by a constant, $|\mathcal{C}(x)| C$, which is independent of $L \geq L_{x}$ and $z \in \mathbb{C}^{+}$.

To bound the second term, $S_{2}$, we use the fact that the Green's function factorizes,

$$
\left\langle\delta_{x},\left(H_{\mathcal{T}_{L}}-E\right)^{-1} \delta_{y}\right\rangle=\left\langle\delta_{x},\left(H_{\mathcal{T}_{L}}-E\right)^{-1} \delta_{v}\right\rangle\left\langle\delta_{w},\left(H_{\mathcal{C}(w)}-E\right)^{-1} \delta_{y}\right\rangle,
$$

where $v$ is the first joint ancestor of $x$ and $y$, and $w$ is that neighbor of $v$ which has the least distance from $y$. We may therefore organize the summation in $S_{2}$ as follows. We sum over the vertices on the unique path in $\mathcal{P}(x) \subset \mathcal{C}$ which connects $x$ and "infinity", cf. Figure1. For each vertex along this path we then collect terms of the form

$$
S(w):=\sum_{y \in \mathcal{C}(w)}\left|\left\langle\delta_{w},\left(H_{\mathcal{C}(w)}-E\right)^{-1} \delta_{y}\right\rangle\right|^{2}
$$


which stem from the $K-1$ neighbors $w$ of $v$, which are not in $\mathcal{P}(x)$. Consequently, the second term in (5.4) is bounded according to

$$
\begin{aligned}
S_{2} & \leq \sum_{v \in \mathcal{P}(x) \cap \mathcal{T}_{L}} \mathbb{E}\left[\left|\left\langle\delta_{x},\left(H_{\mathcal{T}_{L}}-E\right)^{-1} \delta_{v}\right\rangle\right|^{2 s}\left[1+\sum_{\substack{\operatorname{dist}(w, v)=1 \\
w \notin \mathcal{P}(x)}} S(w)^{s}\right]\right] \\
& \leq \sum_{v \in \mathcal{P}(x) \cap \mathcal{T}_{L}} \sum_{\substack{\operatorname{dist}(w, v)=1 \\
w \notin \mathcal{P}(x)}} \mathbb{E}\left[\left|\left\langle\delta_{x},\left(H_{\mathcal{T}_{L}}-E\right)^{-1} \delta_{v}\right\rangle\right|^{4 s}\right]^{1 / 2}\left[1+\left(\mathbb{E}\left[S(w)^{2 s}\right]^{1 / 2}\right)\right] \\
& \leq C(s, K) \sum_{v \in \mathcal{P}(x) \cap \mathcal{T}_{L}}\left(\mathbb{E}\left[K^{2 s \operatorname{dist}(x, v)}\left|\left\langle\delta_{x},\left(H_{\mathcal{T}_{L}}-E\right)^{-1} \delta_{v}\right\rangle\right|^{4 s}\right]\right)^{1 / 2} \\
\times & {\left[1+\left(\mathbb{E}\left[\frac{1}{|C(w)|^{2 s}} S(w)^{2 s}\right]\right)^{1 / 2}\right], }
\end{aligned}
$$

where $C(s, K)<\infty$ is independent of $w$ and $v$, and $w$ is any of the $(K-1)$ neighbors of $v$ with $w \notin \mathcal{P}(x)$. According to Lemma 5.2 below, the last term in the right side of (5.7) is bounded from above by a constant which is independent of $w$. Lemma3.2 then proves that the remaining sum over $v \in \mathcal{P}(x) \cap \mathcal{T}_{L}$ in 5.77 is bounded from above by a constant which is independent of $L \in \mathbb{N}$.

Lemma 5.2. Under assumption A1 for any $s \in(0,1 / 4)$

$$
\sup _{z \in \mathbb{C}^{+}} \sup _{L \in \mathbb{N}} \mathbb{E}\left[\frac{1}{\left|\mathcal{T}_{L}\right|^{s}}\left|\left\langle\delta_{0},\left|H_{\mathcal{T}_{L}}-z\right|^{-2} \delta_{0}\right\rangle\right|^{s}\right]<\infty .
$$

Proof. A combination of (2.12), 2.13) (with $w=1$ ) and 2.11) below yields for all $z \in \mathbb{C}^{+}$and $L \in \mathbb{N}$

$$
\begin{aligned}
\left|\mathcal{T}_{L}\right|^{-1}\left\langle\delta_{0},\left|H_{\mathcal{T}_{L}}-z\right|^{-2} \delta_{0}\right\rangle \leq \operatorname{Im}\langle & \delta_{0}, \\
& \left.\left(H_{\mathcal{T}_{L}}-\operatorname{Re} z-i\left|\mathcal{T}_{L}\right|^{-1}\right)^{-1} \delta_{0}\right\rangle \\
& \times\left(2+\left|\mathcal{T}_{L}\right|^{-2} \operatorname{dist}\left(\sigma\left(H_{\mathcal{T}_{L}}\right), z\right)^{-2}\right) .
\end{aligned}
$$

We now take the fractional-moment and apply the Cauchy-Schwarz inequality. The claim then follows from the fractional-moment bound 3.1) and Wegner's estimate (2.1).

Proof of Theorem 1.3 We pick an arbitrary bounded Borel set $I \subset \mathbb{R}$. By the strong resolvent convergence,

$$
\lim _{L \rightarrow \infty}\left\|\left(H_{\mathcal{T}_{L}}-z\right)^{-1} \delta_{x}-\left(H_{\mathcal{C}}-z\right)^{-1} \delta_{x}\right\|=0
$$

for all $x \in \mathcal{C}$ and all $z \in \mathbb{C}^{+}$, and monotone convergence, it follows from (5.1) that for Lebesgue-almost all $E \in I$

$$
\mathbb{E}\left[\left(\lim _{\eta \downarrow 0}\left\|\left(H_{\mathcal{C}}-E-i \eta\right)^{-1} \delta_{x}\right\|^{2}\right)^{s}\right]<\infty
$$


with the same $s$ as in (5.1). Since the conditional distribution of $\omega_{x}$-conditioned on the sigma-algebra generated by $\left\{\omega_{y}\right\}_{y \neq x}$ - has a bounded density, $\varrho$, the Simon-Wolff localization criterion [SW86, Thm. 8] is thus satisfied and yields the assertion.

\section{The spectra of random operators on single-ended trees}

On the canopy tree $\mathcal{C}$ from any site there is a unique path to infinity, i.e., in the terminology of [Wo00] it is a single-ended tree. The purpose of this section is to clarify that this point in itself implies part of above localization statement, but not all of it. To place Theorem 1.3 in a more general context we prove here that on single-ended trees random operators of the kind considered here have no absolutely continuous spectrum, but singular continuous spectrum can occur (though not in the specific case of the canopy graph).

\subsection{Absence of absolutely continuous spectrum}

The main result of the present subsection is proven for the class of graphs defined next.

Definition 6.1. For a graph $\mathcal{G}$ a backbone $\mathcal{B}$ is a connected path, indexed by either $\mathbb{Z}$, $\mathbb{N}$, or $\{1, \ldots, L\}$, whose deletion transforms $\mathcal{G}$ into a collection of finite disconnected sets. Graphs with a backbone are referred to as backbone graphs.

Not every graph has a backbone, and in case it does, the backbone is not unique (except in the double-ended case) as can be seen by considering the canopy graph. We shall consider below self-adjoint random operators

$$
H_{\mathcal{G}, \mathcal{B}}=A+W+V
$$

acting on the Hilbert space $\ell^{2}(\mathcal{G})$ over a backbone graph, where $A$ denotes the adjacency operator and $W$ stands for an arbitrary multiplication operator. Moreover, $V$ denotes the random multiplication operator which acts only along the backbone with values at sites $x \in \mathcal{B}$ given by random variables $\left\{\omega_{x}\right\}_{x \in \mathcal{B}}$ which we assume to be independent and identically distributed, with a distribution satisfying the following assumption.

Assumption A1': The distribution of the variables $\omega_{x}$ is of bounded density, $\varrho \in L^{\infty}(\mathbb{R})$, and satisfies $\int_{\mathbb{R}}\left(1+\left|\omega_{0}\right|\right)^{2} \varrho\left(\omega_{0}\right)^{2} d \omega_{0}<\infty$.

Note that Assumption A1 with $\tau=2$ implies Assumption A1'.

Theorem 6.1 (Absence of ac spectrum). Assuming A1', for any operator $H_{\mathcal{G}, \mathcal{B}}=$ $A+W+V$ on the Hilbert-space $\ell^{2}(\mathcal{G})$ associated with a backbone graph the absolutely continuous component of the spectrum is empty.

In the proof we shall make use of the following extension of a bound which was proven for one dimensional random operators in the work of Delyon, Kunz, and Souillard [DKS83]. For this result we assume the structure described above, except that it is not necessary for the backbone to extend to infinity. 
Proposition 6.2 (Exponential decay II). On a finite graph $\mathcal{G}$ with a backbone $\mathcal{B}$, let $H_{\mathcal{G}, \mathcal{B}}=A+W+V$ be a random operator of the form described above. Assuming A1' holds, for any bounded interval $I \subset \mathbb{R}$ and $s \in(0,1 / 2)$ there exists $C(s, I)<\infty$ such that for any pair of sites along the backbone $x, y \in \mathcal{B}$ :

$$
\int_{I} \mathbb{E}\left[\left|\left\langle\delta_{x},\left(H_{\mathcal{G}, \mathcal{B}}-E\right)^{-1} \delta_{y}\right\rangle\right|^{s}\right] d E \leq C(s, I) \exp (-s \lambda \operatorname{dist}(x, y))
$$

where (as in [DKS83 Eq. (1.8)])

$$
\lambda:=\inf _{\substack{\eta>0 \\ 40 \eta|\log \eta|<1}}-2 / \log \left(1-\frac{\alpha(\eta)}{25}(1-40 \eta|\log \eta|)^{2}\right),
$$

with $\alpha(\eta):=1-\sup _{|\xi|>\eta}|\hat{\varrho}(\xi)|$, and $\hat{\varrho}$ the Fourier transform of the single-site density.

Although it is not stated there in the above form, this exponential bound readily follows from the one-dimensional analysis of [DKS83]. We present this reduction in Appendix ??. Assuming Proposition 6.2 we turn now to the derivation of the main result of this section.

Proof of Theorem 6.1 We shall give the proof for $\mathcal{B}$ single-ended; the case of a doubleended backbone follows similarly and, in case $\mathcal{B}$ is finite there is nothing to show.

Let $x_{0} \in \mathcal{B}$ be arbitrary and pick $x_{L} \in \mathcal{B}$ in the direction towards infinity with $\operatorname{dist}\left(x_{0}, x_{L}\right)=L$. By removing the bond between $x_{L}$ and $x_{L+1}$, we cut $\mathcal{G}$ and its backbone into finite parts $\mathcal{G}_{L}, \mathcal{B}_{L}$ and the infinite remainders. Let $H_{\mathcal{G}_{L}, \mathcal{B}_{L}}$ denote the restriction of $H_{\mathcal{G}, \mathcal{B}}$ to $\ell^{2}\left(\mathcal{G}_{L}\right)$.

The resolvent identity and the fact that $\operatorname{Im}\left\langle\delta_{x_{0}},\left(H_{\mathcal{G}_{L}, \mathcal{B}_{L}}-E\right)^{-1} \delta_{x_{0}}\right\rangle=0$ for almost all $E \in \mathbb{R}$ implies

$$
\begin{aligned}
\operatorname{Im}\left\langle\delta_{x_{0}},\left(H_{\mathcal{G}, \mathcal{B}}-E-i 0\right)^{-1} \delta_{x_{0}}\right\rangle \leq & \left|\left\langle\delta_{x_{0}},\left(H_{\mathcal{G}_{L}, \mathcal{B}_{L}}-E\right)^{-1} \delta_{x_{L}}\right\rangle\right|^{2} \\
& \times\left|\left\langle\delta_{x_{L+1}},\left(H_{\mathcal{G}, \mathcal{B}}-E-i 0\right)^{-1} \delta_{x_{L+1}}\right\rangle\right|
\end{aligned}
$$

Since the ac component of the spectral measure of $H_{\mathcal{G}, \mathcal{B}}$ associated with $\delta_{x_{0}}$ is supported on those $E \in \mathbb{R}$ for which the left-hand side is finite and strictly positive, it remains to show that the right-hand side converges as $L \rightarrow \infty$ to zero in distribution with respect to the product of the probability measure associated with $\left\{\omega_{x}\right\}$ and Lebesgue measure for $E \in I$, where $I \subset \mathbb{R}$ is an arbitrary bounded interval. For a proof of the latter we note that the second term on the right-hand side of 6.4) is seen to be bounded in probability using, for example, a fractional moment estimate, cf. (3.1). Moreover, Proposition 6.2 implies that the first term converges to zero in distribution with respect to the above product measure.

\subsection{Appearance of singular continuous spectrum}

We shall now focus on backbone graphs which are obtained by decorating an infinite path $\mathcal{B}$ with trees, as in Figure 2. The canopy graph is within this class but our main result applies to graphs where the trees attached to the line grow at a much faster rate. 
The goal is to prove that there are single-ended trees $\mathcal{G}$ for which random operators of the form

$$
H_{\mathcal{G}, \mathcal{B}}=A+V
$$

have a singular continuous component in their spectrum. Here and in the following, we will assume that $V$ is the multiplication operator corresponding to independent and identically distributed random variables $\left\{\omega_{x}\right\}_{x \in \mathcal{G}}$ on the whole tree.

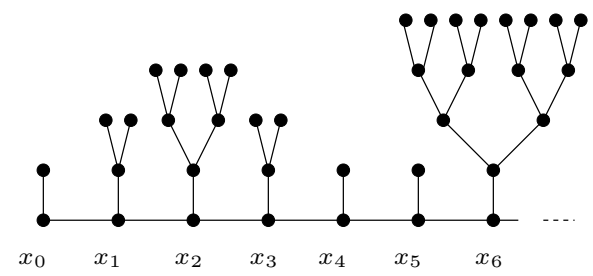

Figure 2: Example of the construction of a singleended tree $\mathcal{G}$ obtained by gluing finite trees to the sites of a backbone.

Theorem 6.3 (Singular continuous spectrum). Fix a bounded, open interval $I \subset$ $\Sigma_{\text {cont }}\left(H_{\mathcal{T}}\right)$ where $H_{\mathcal{T}}=A+V$ with random variables satisfying A1. Then there exist a single-ended tree $\mathcal{G}$ for which $H_{\mathcal{G}, \mathcal{B}}=A+V$ has singular continuous spectrum on $I$.

Two remarks apply:

1. The existence of continuous spectrum, $\Sigma_{\text {cont }}\left(H_{\mathcal{T}}\right) \neq \emptyset$ for the random operator $H_{\mathcal{T}}=A+V$ on the infinite rooted regular tree $\mathcal{T}$ for small disorder is ensured by [K195, K198, ASW06a, FHS07].

2. It has been noted before that random operators with decaying potential [D85] or the Laplacian on certain (tree) graphs [Si96, Br06] may exhibit singular continuous spectrum.

The construction of trees $\mathcal{G}$ in Theorem 6.3 is based on the following observation.

Lemma 6.4. Assume $\mathrm{A} 1$ and let $I \subset \Sigma_{\text {cont }}\left(H_{\mathcal{T}}\right)$ be a bounded Borel set and $s \in$ $(0, \tau / 2]$. Then

$$
\lim _{L \rightarrow \infty} \int_{I} \mathbb{E}\left[\left\langle\delta_{0},\left(H_{\mathcal{T}_{L}}-E\right)^{-2} \delta_{0}\right\rangle^{-s}\right] d E=0 .
$$

Proof. We pick $\eta>0$. The spectral theorem implies that for every $L \in \mathbb{N}$

$$
\begin{aligned}
\left\langle\delta_{0},\left(H_{\mathcal{T}_{L}}-E\right)^{-2} \delta_{0}\right\rangle^{-1} & \leq\left\langle\delta_{0},\left[\left(H_{\mathcal{T}_{L}}-E\right)^{2}+\eta^{2}\right]^{-1} \delta_{0}\right\rangle^{-1} \\
& \leq\left\langle\delta_{0}\left(H_{\mathcal{T}_{L}}-E\right)^{2} \delta_{0}\right\rangle+\eta^{2} \leq 4 K+\omega_{0}^{2}+E^{2}+\eta^{2}
\end{aligned}
$$

Using the dominated convergence theorem and strong resolvent convergence we thus conclude

$$
\begin{aligned}
\lim _{L \rightarrow \infty} & \int_{I} \mathbb{E}\left[\left\langle\delta_{0},\left(H_{\mathcal{T}_{L}}-E\right)^{-2} \delta_{0}\right\rangle^{-s}\right] d E \\
& \leq \int_{I} \mathbb{E}\left[\left\langle\delta_{0},\left[\left(H_{\mathcal{T}}-E\right)^{2}+\eta^{2}\right]^{-1} \delta_{0}\right\rangle^{-s}\right] d E
\end{aligned}
$$


The claim follows with the help of the the dominated convergence theorem from the fact that for Lebesgue almost all $E \in \Sigma_{\text {cont }}\left(H_{\mathcal{T}}\right)$

$$
\lim _{\eta \downarrow 0}\left\langle\delta_{0},\left[\left(H_{\mathcal{T}}-E\right)^{2}+\eta^{2}\right]^{-1} \delta_{0}\right\rangle^{-1}=0 .
$$

The proof of Theorem 6.3 is also based on the following Simon-Wolff type criterion, which may be deduced from [RSS94].

Proposition 6.5 (cf. [RSS94]). Let $H$ be a random operator on the Hilbert space over a graph, with iid random potential whose single site distribution is ac and bounded. Assume there is a Borel set I such that

1. H has no absolutely continuous spectrum in I.

2. for Lebesgue almost every $E \in I$ almost surely the Green function is not square summable:

$$
\lim _{\eta \downarrow 0}\left\langle\delta_{x},\left[(H-E)^{2}+\eta^{2}\right]^{-1} \delta_{x}\right\rangle^{-1}=0 .
$$

Then, in the space for which $\delta_{x}$ is a cyclic vector, almost surely $H$ has only singular continuous spectrum in I.

Finally, our construction is also based on the following lower bound on the decay of the Green function of single-ended graphs $\mathcal{G}$ along the backbone $\mathcal{B}$. It is important for us that the decay rate is controlled independently of the depth of the trees glued to $\mathcal{B}$.

Lemma 6.6. Assume $\mathbf{A 1}$ and let $E_{0}>0$ and $s \in(0, \min \{\tau, 1 / 2\}]$. There exist some constant $C\left(s, E_{0}\right)<\infty$ such that for all $x_{0}, x \in \mathcal{B}$

$$
\begin{gathered}
\sup _{|E| \leq E_{0}} \sup _{\eta \in(0,1)} \mathbb{E}\left[\left|\left\langle\delta_{x_{0}},\left(H_{\mathcal{G}, \mathcal{B}}-E-i \eta\right) \delta_{x}\right\rangle\right|^{-s} \mid\left\{\omega_{x}\right\}_{x \notin\{y: \operatorname{dist}(y, \mathcal{B}) \leq 1\}}\right] \\
\leq C\left(s, E_{0}\right) e^{\lambda\left(s, E_{0}\right) \operatorname{dist}\left(x_{0}, x\right)} .
\end{gathered}
$$

Here

$$
\lambda\left(s, E_{0}\right):=1+\log \left(1+E_{0}+\int\left|\omega_{0}\right|^{s} \varrho\left(\omega_{0}\right) d \omega_{0}+K^{\prime} C_{s}\right),
$$

where $K^{\prime}$ is the maximal number of vertices neighboring $\mathcal{B}$, and $C_{s}$ is the constant appearing in 3.1 .

Proof. Similarly as in (3.5) (cf. [K198]) we factorize the Green function into a product,

$$
\begin{array}{r}
\left\langle\delta_{x_{0}},\left(H_{\mathcal{G}, \mathcal{B}}-E-i \eta\right)^{-1} \delta_{x}\right\rangle=\prod_{j=0}^{\operatorname{dist}\left(x_{0}, x\right)} \Gamma_{j} \\
\text { with } \quad \Gamma_{j}:=\left\langle\delta_{x_{j}},\left(H_{\mathcal{G}\left(x_{j}\right)}-E\right)^{-1} \delta_{x_{j}}\right\rangle .
\end{array}
$$


Here $x_{0}, x_{1}, \ldots, x_{\operatorname{dist}(0, x)}:=x$ are the vertices on $\mathcal{B}$ connecting $x_{0}$ with $x$. Moreover, $\mathcal{G}\left(x_{j}\right)$ is that infinite subtree of $\mathcal{G}$ which is forward to $x_{j}$ in the direction away from $x_{0}$. The factors in (6.13) satisfy the following relation (cf. [K198])

$$
\begin{aligned}
\Gamma_{j}^{-1}= & V_{x_{j}}-E-i \eta-\Gamma_{j+1}-G_{j} \\
& \text { where } G_{j}:=\sum_{\substack{\operatorname{dist}\left(w, x_{j}\right)=1 \\
w \notin \mathcal{B}}}\left\langle\delta_{w},\left(H_{\mathcal{G}(w)}-E\right)^{-1} \delta_{w}\right\rangle,
\end{aligned}
$$

and the sum is over all neighboring vertices of $x_{j}$ which are not on $\mathcal{B}$ and each term involves the finite subtree tree $\mathcal{G}(w)$ which is rooted at $w$ and extends away from the backbone.

We now integrate the product in 6.13 step by step starting with estimating the conditional expectation, conditioning on all random variables aside from $x_{0}$ and its neighbors which do not belong to $\mathcal{P}\left(x_{0}\right)$,

$$
\begin{aligned}
& \mathbb{E}\left[\left|\Gamma_{0}\right|^{-s} \mid\left\{\omega_{x_{0}}, \omega_{w}\right\}_{\left\{w \notin \mathcal{B}: \operatorname{dist}\left(w, x_{0}\right)=1\right\}}^{c}\right] \\
& \leq \int\left|\omega_{0}\right|^{s} \varrho\left(\omega_{0}\right) d \omega_{0}+|E|+\eta+\left|\Gamma_{1}\right|^{-s}+K^{\prime} C_{s} \\
& \leq \exp \left(\lambda\left(s, E_{0}\right)-1\right)+\left|\Gamma_{1}\right|^{-s} .
\end{aligned}
$$

Here the last factor in the second line stems from estimating the expectation of the terms contributing to $G_{0}$ using 3.1]. Iterating this bound yields the result.

Proof of Theorem 6.3 Let $E_{I}=\max \{|E|: E \in I\}$ and $\tau^{\prime}:=\min \{\tau, 1 / 2\} / 2$. We define a sequence of finite regular trees $\mathcal{T}_{L_{n}}$ through the requirement

$$
\int_{I} \mathbb{E}\left[\left\langle\delta_{0},\left(H_{\mathcal{T}_{L_{n}}}-E\right)^{-2} \delta_{0}\right\rangle^{-\tau^{\prime}}\right] d E \leq \exp \left(-2 \lambda\left(2 \tau^{\prime}, E_{I}\right) n\right)
$$

for every $n \in \mathbb{N}_{0}$, where $\lambda\left(\tau^{\prime} / 4, E_{I}\right)$ is the constant appearing in 6.12). Note that such a sequence exists thanks to Lemma 6.4 .

Given a half-infinite line $\mathcal{B}$ we glue to every vertex $x_{n} \in \mathcal{B}$ with $\operatorname{dist}\left(x_{0}, x_{n}\right)=n$ another edge which connects $x_{n}$ with the root of the tree $\mathcal{T}_{L_{n}} ;$ cf. Figure 2

To conclude that the spectral measure of $H_{\mathcal{G}, \mathcal{B}}$ associated with $\delta_{x_{0}}$ is purely singular continuous in $I$, we use Proposition 6.5 and first note that for any $L \in \mathbb{N}$ and Lebesguealmost every $E \in \mathbb{R}$

$$
\begin{aligned}
& \liminf _{\eta \downarrow 0}\left\langle\delta_{x_{0}},\left[\left(H_{\mathcal{G}, \mathcal{B}}-E\right)^{2}+\eta^{2}\right]^{-1} \delta_{x_{0}}\right\rangle \\
& \geq \liminf _{\eta \downarrow 0} \sum_{x \in \mathcal{B}_{L}}\left|\left\langle\delta_{x_{0}},\left(H_{\mathcal{G}, \mathcal{B}}-E-i \eta\right)^{-1} \delta_{x}\right\rangle\right|^{2} \\
& =\sum_{n=0}^{L}\left|\left\langle\delta_{x_{0}},\left(H_{\mathcal{G}, \mathcal{B}}-E-i 0\right)^{-1} \delta_{x_{n}}\right\rangle\right|^{2}(1+S(n)) \\
& \quad \text { where } \quad S(n):=\left\langle\delta_{0},\left(H_{\mathcal{T}_{L_{n}}}-E\right)^{-2} \delta_{0}\right\rangle .
\end{aligned}
$$


We now average an inverse power of the above quantity and integrate over $I$. Jensen's inequality thus yields

$$
\begin{aligned}
& \int_{I} \mathbb{E}\left[\underset{\eta \downarrow 0}{\limsup }\left\langle\delta_{x_{0}},\left[\left(H_{\mathcal{G}, \mathcal{B}}-E\right)^{2}+\eta^{2}\right]^{-1} \delta_{x_{0}}\right\rangle^{-\tau^{\prime} / 2}\right] d E \\
& \leq \frac{1}{L^{\tau^{\prime}+1}} \sum_{n=0}^{L} \int_{I} \mathbb{E}\left[\left|\left\langle\delta_{x_{0}},\left(H_{\mathcal{G}, \mathcal{B}}-E-i 0\right)^{-1} \delta_{x_{n}}\right\rangle\right|^{-\tau^{\prime}} S(n)^{-\tau^{\prime} / 2}\right] d E .
\end{aligned}
$$

The Cauchy-Schwarz inequality together with 6.16 and Lemma 6.6 imply that the right-hand side in 6.18 is bounded from above by

$$
\frac{1}{L^{\tau^{\prime}+1}} C\left(2 \tau^{\prime}, E_{0}\right)^{1 / 2} \sum_{n=0}^{L} \exp \left(-\lambda\left(2 \tau^{\prime}, E_{0}\right) \frac{n}{2}\right),
$$

which converges to zero as $L \rightarrow \infty$. This yields the claimed result.

The lengths of the regular trees $\mathcal{T}_{L_{n}}$ are defined via (6.16) in a rather indirect way. In particular, no estimates are given. To answer the question about the minimal growth of $L_{n}$ as $n \rightarrow \infty$, which is sufficient for the production of singular continuous spectrum, one needs to estimate the growth of the quantity $\left\langle\delta_{0},\left(H_{\mathcal{T}_{L}}-E\right)^{-2} \delta_{0}\right\rangle$ as $L \rightarrow \infty$ for $E \in \Sigma_{\text {cont }}\left(H_{\mathcal{T}}\right)$.

\section{Appendix}

For completeness, in the following appendix sections we shall briefly sketch proofs of some spectral properties of the canopy graph which are of relevance to our discussion and which are derived by arguments which are already in the literature. We also add some observations and discussion.

\section{A Some basic properties of the canopy operator}

\section{A.1 Existence of the canopy density of states measure}

Following is a brief sketch of the proof of Theorem 1.2

Proof of Theorem 1.2 We embed $\mathcal{T}_{L}$ into $\mathcal{C}$ so that $\partial \mathcal{T}_{L} \subset \partial \mathcal{C}$. The trace in 1.10) can be decomposed into contributions from layers with a fixed distance to the outer 
boundary,

$$
\begin{aligned}
&\left|\mathcal{T}_{L}\right|^{-1} \operatorname{Tr} F\left(H_{\mathcal{T}_{L}}\right)=\frac{K-1}{K} \sum_{n=0}^{L} K^{-n} T_{n, L}(F) \\
& \text { where } T_{n, L}(F):=K^{n+1-L} \sum_{x: \operatorname{dist}\left(x, \partial \mathcal{T}_{L}\right)=n}\left\langle\delta_{x}, F\left(H_{\mathcal{T}_{L}}\right) \delta_{x}\right\rangle
\end{aligned}
$$

Each contribution $T_{n, L}(F)$ is normalized to one for $F=1$ and, more generally, $T_{n, L}(F) \leq\|F\|_{\infty}$. Thanks to dominated convergence, it is therefore enough to prove the following almost-sure convergence for each $n \in \mathbb{N}_{0}$

$$
\lim _{L \rightarrow \infty} T_{n, L}(F)=\mathbb{E}\left[\left\langle\delta_{x_{n}}, F\left(H_{\mathcal{C}}\right) \delta_{x_{n}}\right\rangle\right],
$$

where $x_{n} \in \mathcal{C}$ is an arbitrary vertex with $\operatorname{dist}\left(x_{n}, \partial \mathcal{C}\right)=n$, cf. Figure 1 .

The proof of A.2 boils down to the Birkhoff-Khintchin ergodic theorem [Ka02] and an approximation argument. Since the functions $\varphi_{z}=(\cdot-z)^{-1}$ with $z \in \mathbb{C}^{+}$ are dense in $C_{b}(\mathbb{R})$ and the linear functionals in both sides of $(\mathrm{A} .2)$ are (uniformly) continuous on $C_{b}(\mathbb{R})$, it is sufficient to prove $\mathrm{A}$.2 for $F=\varphi_{z}$.

By truncating $\mathcal{T}_{L}$ at a layer $n+L_{0}$ below the outer boundary, we may approximate the sum $T_{n, L}\left(\varphi_{z}\right)$ by $K^{L-n-L_{0}}$ stochastically independent terms of the form

$$
\frac{1}{K^{L_{0}}} \sum_{\operatorname{dist}(0, y)=L_{0}}\left\langle\delta_{y}, \varphi_{z}\left(H_{\mathcal{T}_{n+L_{0}}}\right) \delta_{y}\right\rangle .
$$

The approximation error can be kept arbitrarily small by taking $L_{0} \in \mathbb{N}$ large. The approximating average of $K^{L-n-L_{0}}$ stochastically independent terms satisfies the assumptions of the Birkhoff-Khintchin ergodic theorem for iid random variables. As $L \rightarrow \infty$, it therefore converges almost surely to

$$
\mathbb{E}\left[\frac{1}{K^{L_{0}}} \sum_{\operatorname{dist}(0, y)=L_{0}}\left\langle\delta_{y}, \varphi_{z}\left(H_{\mathcal{T}_{n+L_{0}}}\right) \delta_{y}\right\rangle\right]=\mathbb{E}\left[\left\langle\delta_{x_{n}}, \varphi_{z}\left(H_{\mathcal{T}_{n+L_{0}}}\right) \delta_{x_{n}}\right\rangle\right],
$$

where $x_{n}$ is an arbitrary vertex in the $n$th layer below the surface $\partial \mathcal{T}_{n+L_{0}}$. Taking $L_{0} \rightarrow \infty$, the last term converges to the right side in $\mathrm{A.2}$ by the dominated convergence theorem.

The standard Wegner estimate allows to conclude some regularity of $n_{\mathcal{C}}$.

Lemma A.1. Under the assumption A1 the canopy density of states measure $n_{\mathcal{C}}$ is absolutely continuous, with bounded density satisfying

$$
d_{\mathcal{C}}(E)=\frac{n_{\mathcal{C}}(d E)}{d E} \leq\|\varrho\|_{\infty} .
$$

Proof. This readily follows from the definition of the density of states measure (1.10) and the Wegner estimate 2.1. 


\section{A.2 Spectrum of the adjacency operator on the canopy graph}

We will now give a brief sketch of the proof of the following assertion:

Proposition A.2 (cf. [AF00]). The spectrum of of the adjacency operator with boundary condition $A+B$ on $\ell^{2}(\mathcal{C})$ consists of infinitely degenerate eigenvalues coinciding with the union of all eigenvalues of the adjacency operator (with constant boundary condition $b \in \mathbb{R})$ on $\ell^{2}(\{1,2, \ldots, n\})$ with $n \in \mathbb{N}$ arbitrary. The corresponding eigenfunctions are compactly supported.

Proof. The basic construction is simplest to describe for $K=2$. In that case, for each eigenfunction $\psi$ of the adjacency operator on $\ell^{2}(\{1,2, \ldots, n\})$ eigenfunctions can be constructed on the canopy graph which are supported on the forward trees corresponding to any site $x$ which is at distance $n+1$ from the outer boundary $\partial \mathcal{C}$. The functions are defined so they are antisymmetric with respect to the exchange of the two forward trees of length $n$ which lie between $x$ and the boundary $\partial \mathcal{C}$, and on each of the two subtrees are given by a radially 'fanned out' versions of $\psi$ (cf. ASW06a, Proof of Prop. A.1]). It is easy to verify that the construction yields a complete orthonormal collection of eigenfunctions.

To determine the spectrum of $A+B$ on $\ell^{2}(\mathcal{C})$ with a general $K$ one may use analogously to [AF00] - a decomposition of the Hilbert space into invariant subspaces:

$$
\ell^{2}(\mathcal{C})=\bigoplus_{x \in \mathcal{C}} \mathcal{Q}_{x}, \quad \text { with } \quad \mathcal{Q}_{x}:=\left(\bigoplus_{\substack{y \in \mathcal{C}(x) \\ \operatorname{dist}(x, y)=1}} \mathcal{S}_{y}\right) \ominus \mathcal{S}_{x}
$$

where $\mathcal{S}_{x}$ denotes the subspace of symmetric functions on the forward subtree $\mathcal{C}(x)$, cf. (5.3):

$$
\mathcal{S}_{x}:=\left\{\psi \in \ell^{2}(\mathcal{C}): \begin{array}{l}
y \mapsto\left\langle\delta_{y}, \psi\right\rangle \text { is supported on } \mathcal{C}(x) \\
\text { and constant on each generation of } \mathcal{C}(x)
\end{array}\right\} .
$$

The orthogonal decomposition A.6 reduces the operator $A+B$ on $\ell^{2}(\mathcal{C})$ to an orthogonal sum of operators on $\mathcal{Q}_{x}$, each of which is unitarily equivalent to the orthogonal sum of $K-1$ operators on $\mathcal{S}_{y}$ where $y$ is one of the forward neighbors of $x$. In turn, each operator on $\mathcal{S}_{y}$ is unitary equivalent to the adjacency operator (with constant boundary condition $b \in \mathbb{R})$ on the Hilbert space $\ell^{2}(\{1,2, \ldots, \operatorname{dist}(y, \partial \mathcal{C})\})$.

As an aside, we note that other examples of discrete operators with finitely supported eigenfunctions can be found in [GZ01, DS01].

\section{B Negligibility in probability of the spectral measure within the singular spectrum}

It may be of some interest to observe that, as is proven below, the condition which by Theorem 2.2 implies Poisson statistics holds throughout the singular spectrum of the 
infinite-volume operator. To avoid confusion, let us note that although the reference here is to the spectral measure at the root, the infinity divisibility which this implies is of the spectral measure whose density is given by the canopy dos, weighted as in (1.9). This observation is not used for our main result since we establish the negligibility in probability thorough another mechanism, which is valid throughout the entire spectrum.

The relevant statement is valid not only in the tree setup and is based on the following measure theoretic statement.

Theorem B.1. Suppose the operator $H_{\mathcal{T}}$ has almost surely only singular spectrum in a given Borel set I. Then for Lebesgue-almost all $E \in I$ the condition in Definition 2.1 is satisfied.

Proof. As $L \rightarrow \infty$ the spectral measure $\sigma_{x, L}$ converges vaguely to $\left\langle\delta_{x}, P .\left(H_{\mathcal{T}}\right) \delta_{x}\right\rangle$, which is finite and purely singular on $I$. The subsequent lemma thus implies that $\mathbb{P}$ almost surely for every $\varepsilon>0$ and $w>0$

$$
\left|\limsup _{L \rightarrow \infty}\left\{E \in I:\left|\mathcal{T}_{L}\right| \sigma_{x, L}\left(E+\left|\mathcal{T}_{L}\right|^{-1}(-w, w)\right)>\varepsilon\right\}\right|=0 .
$$

By Fubini-Tonelli's theorem this implies that for every $\varepsilon>0$ and every $w>0$ there exists a subset $J(\varepsilon, w) \subset \Sigma_{\operatorname{sing}}\left(H_{\mathcal{T}}\right)$ of full Lebesgue measure such that for all $E \in$ $J(\varepsilon, w)$

$$
\begin{aligned}
\lim _{L \rightarrow \infty} \mathbb{P}\left(\left\{\left|\mathcal{T}_{L}\right| \sigma_{x, L}\left(E+\left|\mathcal{T}_{L}\right|^{-1}(-w, w)\right)>\varepsilon\right\}\right) \\
\quad \leq \mathbb{P}\left(\limsup _{L \rightarrow \infty}\left\{\left|\mathcal{T}_{L}\right| \sigma_{x, L}\left(E+\left|\mathcal{T}_{L}\right|^{-1}(-w, w)\right)>\varepsilon\right\}\right)=0 .
\end{aligned}
$$

Since the event in the right-hand side is monotone in both $\varepsilon$ and $w$, we may pick any two monotone sequences $\epsilon_{n} \rightarrow 0$ and $w_{m} \rightarrow \infty$ and define $J:=\bigcap_{n, m} J\left(\varepsilon_{n}, w_{m}\right)$, a set of full Lebesgue measure, on which the claimed convergence (2.5) holds for all $\varepsilon>$ and $w>0$.

Following is a rather general observation for singular measures.

Lemma B.2. Let $\sigma$ be a purely singular measure on $I \subset \mathbb{R}$, suppose that $\lim _{n \rightarrow \infty} \sigma_{n}=$ $\sigma$ vaguely, and let $\left\{\xi_{n}\right\}_{n=0}^{\infty}$ be a null sequence. Then for every $\varepsilon>0$ and $w>0$ the sequence of sets

$$
A_{n}(\varepsilon, w):=\left\{E \in I: \sigma_{n}\left(E-w \xi_{n}, E+w \xi_{n}\right)>\varepsilon \xi_{n}\right\}
$$

satisfies

$$
\left|\limsup _{n \rightarrow \infty} A_{n}(\varepsilon, w)\right|=\left|\bigcup_{n=0}^{\infty} \bigcap_{m=n}^{\infty} A_{n}(\varepsilon, w)\right|=0 .
$$

Proof. We prove the assertion by contradiction. Suppose there exists $\varepsilon>0, w>0$, $M \in \mathbb{N}$ such that

$$
\left|\bigcap_{m=M}^{\infty} A_{n}(\varepsilon, w)\right|>0 .
$$


This implies that there exists an open ball $B \subset \bigcap_{m=M}^{\infty} A_{n}(\varepsilon, w)$. By assumption $\sigma$ is purely singular on this ball, such that for every $\delta>0$ there exists a finite collection of disjoint closed intervals $\left\{I_{k}^{\delta}\right\}_{k=1}^{N_{\delta}}$, each of which is contained in $B$, such that [Ka02]

$$
\left|B \backslash \bigcup_{k=1}^{N_{\delta}} I_{k}^{\delta}\right|<\delta \text { and } \sigma\left(\bigcup_{k=1}^{N_{\delta}} I_{k}^{\delta}\right)<\delta .
$$

Since the above intervals are closed, vague convergence implies

$$
\sigma\left(\bigcup_{k=1}^{N_{\delta}} I_{k}^{\delta}\right) \geq \limsup _{n \rightarrow \infty} \sigma_{n}\left(\bigcup_{k=1}^{N_{\delta}} I_{k}^{\delta}\right)=\sum_{k=1}^{N_{\delta}} \limsup _{n \rightarrow \infty} \sigma_{n}\left(I_{k}^{\delta}\right) .
$$

Since the intervals are contained in $\bigcap_{m=M}^{\infty} A_{n}(\varepsilon, w)$, it follows by a covering argument that for every $\delta>0$ and $k \in\left\{1, \ldots, N_{\delta}\right\}$

$$
\limsup _{n \rightarrow \infty} \sigma_{n}\left(I_{k}^{\delta}\right) \geq \frac{\varepsilon}{2}\left|I_{k}^{\delta}\right| \text {. }
$$

Inserting this inequality in $\mathrm{B.6}$, we thus obtain $\delta>\frac{\varepsilon}{2}(|B|-\delta)$, which yields a contradiction for $\delta$ small enough.

\section{A decorated Delyon-Kunz-Souillard bound}

It is rather generally true that eigenfunctions of one-dimensional random operators decay exponentially. In particular, Delyon, Kunz and Souillard [DKS83] (see also [KS80]) presented a proof of localization which does not require translational covariance. Their result can be used in a fairly straightforward way to imply Proposition 6.2, which asserts a generalization of their result to exponential decay of the Green function on graphs which arise from an arbitrary decorations of a line with finite graphs. The statement which is more directly related to the result of [KS80] is the exponential decay of the eigenfunction correlation. We shall first prove that and then use it to derive Proposition 6.2

Proposition C.1. In the setting of Proposition 6.2 there exists $C<\infty$ such that for any bounded interval $I \subset \mathbb{R}$ and any $x, y \in \mathcal{B}$ :

$$
\mathbb{E}\left[\sum_{E \in I \cap \operatorname{spec} H_{\mathcal{G}, \mathcal{B}}}\left|\psi_{E}(x)\right|\left|\psi_{E}(y)\right|\right] d E \leq C|I| \exp (-\lambda \operatorname{dist}(x, y))
$$

with $\lambda>0$ as in 6.3, which is independent of $I$.

Proof. We proceed by relating Proposition C.1 with a result in [DKS83]. In essence, the point is that for a graph $\mathcal{G}$ with random potential along the backbone $\mathcal{B}$, the restriction of an eigenfunction to $\mathcal{B}$ coincides with an eigenfunction of a one dimensional operator for which the rest of $\mathcal{G}$ provides an energy dependent potential. The analysis of [DKS83] is carried pointwise in energy, and hence it is applicable also to the backbone graphs which are considered here. 
Let us start by recalling a change of variable formula, for which $\mathcal{B}$ can be any finite subgraph of a graph $\mathcal{G}$. Let $T$ be an arbitrary self-adjoint operator in $\ell^{2}(\mathcal{G})$ and suppose that $V$ a random multiplication operator whose potential variables $\left\{\omega_{x}\right\}_{x \in \mathcal{B}}$ are independently distributed with densities $\varrho_{x} \in L^{1}(\mathbb{R})$. Moreover, let $\psi_{E}$ be the $\ell^{2}(\mathcal{G})$-normalized eigenfunctions of $H_{\mathcal{G}, \mathcal{B}}=T+V$ at eigenvalues $E \in \operatorname{spec} H_{\mathcal{G}, \mathcal{B}}$. The the following relation holds between probability averages:

$$
\begin{aligned}
\int \prod_{x \in \mathcal{B}} \varrho_{x}\left(\omega_{x}\right) d \omega_{x} \sum_{E \in \operatorname{spec}\left(H_{\mathcal{G}, \mathcal{B}}\right)}\left|\psi_{E}\left(x_{0}\right)\right|^{2}(\cdots) \\
=\int \prod_{x \neq x_{0}} \varrho_{x}\left(\omega_{x}\right) d \omega_{x} \int d E \varrho_{x_{0}}\left(V_{x_{0}}\left(E,\left\{\omega_{x}\right\}_{x \neq x_{0}}\right)\right)(\cdots)
\end{aligned}
$$

where in the last integral $V_{x_{0}}$ is regarded as a function of $E$ and $\left\{\omega_{x}\right\}_{x \neq x_{0}}$, defined so that $E \in \operatorname{spec} H_{\mathcal{G}, \mathcal{B}}$, i.e.,

$$
V_{x_{0}}\left(E,\left\{\omega_{x}\right\}_{x \neq x_{0}}\right):=-\left\langle\delta_{x_{0}},\left(H_{\mathcal{G}, \mathcal{B}}^{(0)}-E\right)^{-1} \delta_{x_{0}}\right\rangle^{-1}
$$

where $H_{\mathcal{G}, \mathcal{B}}^{(0)}$ is a precursor of $H_{\mathcal{G}, \mathcal{B}}$ with $\omega_{x_{0}}=0$.

The relation between $H_{\mathcal{G}, \mathcal{B}}^{(0)}$ and $H_{\mathcal{G}, \mathcal{B}}$ is such that the Green function of the former appears as the eigenfunction of the latter when $V_{x_{0}}$ is chosen as above, and in particular:

$$
\frac{\psi_{E}(x)}{\psi_{E}\left(x_{0}\right)}=\frac{\left\langle\delta_{x_{0}},\left(H_{\mathcal{G}, \mathcal{B}}-E\right)^{-1} \delta_{x}\right\rangle}{\left\langle\delta_{x_{0}},\left(H_{\mathcal{G}, \mathcal{B}}-E\right)^{-1} \delta_{x_{0}}\right\rangle} .
$$

Furthermore, the ratio on the right does not depend on the value of $V_{x_{0}}$.

For the correlation of eigenfunctions at energies in a Borel set $I \subset \mathbb{R}$, which is the quantity of interest for us, the above considerations yield:

$$
\mathbb{E}\left[\sum_{E \in \operatorname{spec}\left(H_{\mathcal{G}, \mathcal{B}}\right) \cap I}\left|\psi_{E}\left(x_{0}\right)\right|\left|\psi_{E}(x)\right|\right]=\int_{I} S\left(x_{0}, x ; E\right) d E .
$$

with

$S\left(x_{0}, x ; E\right):=\int\left|\frac{\left\langle\delta_{x_{0}},\left(H_{\mathcal{G}}-E\right)^{-1} \delta_{x}\right\rangle}{\left\langle\delta_{x_{0}},\left(H_{\mathcal{G}}-E\right)^{-1} \delta_{x_{0}}\right\rangle}\right| \varrho_{x_{0}}\left(V_{x_{0}}\left(E,\left\{\omega_{y}\right\}_{y \neq x_{0}}\right)\right) \prod_{y \neq x_{0}} \varrho_{y}\left(\omega_{y}\right) d \omega_{y}$.

This representation is obtained by averaging $\left|\frac{\psi_{E}(x)}{\psi_{E}\left(x_{0}\right)}\right| 1_{I}(E)$ with respect to the probability measure in (C.2).

In case of a backbone graph (in the sense of Definition 6.1) one may now further change variables in the integral in (C.6) and introduce the Riccatti variables associated with $x \in \mathcal{B} \backslash\left\{x_{0}\right\}$

$$
r_{x}:= \begin{cases}\frac{\psi_{E}(x-1)}{\psi_{E}(x)}, & x>x_{0}, \\ \frac{\psi_{E}(x+1)}{\psi_{E}(x)}, & x<x_{0} .\end{cases}
$$


For $H_{\mathcal{G}, \mathcal{B}}=A+W+V$ these variables are related to the random variables $\left\{\omega_{x}\right\}_{x \neq x_{0}}$ through the Schrödinger equation $\omega_{x}=E-W_{x}-\frac{\psi_{E}(x+1)}{\psi_{E}(x)}-\frac{\psi_{E}(x-1)}{\psi_{E}(x)}$. This implies that

$$
\begin{aligned}
& S\left(x_{0}, x ; E\right)=\int\left(\left|r_{1}\right|^{-1}\left|r_{2}\right|^{-1} \cdots\left|r_{x}\right|^{-1}\right) \varrho_{x_{0}}\left(E-W_{x_{0}}-r_{-1}^{-1}-r_{1}^{-1}\right) \\
& \times \prod_{y>x_{0}} \varrho_{y}\left(E-W_{y}-r_{y}-r_{y+1}^{-1}\right) d \omega_{y} \prod_{y<x_{0}} \varrho_{y}\left(E-W_{y}-r_{y}-r_{y-1}^{-1}\right) d \omega_{y} .
\end{aligned}
$$

The above integral is identical to that in [DKS83, Eq. (2.11)]. It is proven there for the case of interest to us, with the random identically distributed, that there exists $C<\infty$ such that for any $E \in I$

$$
S\left(x_{0}, x ; E\right) \leq C e^{-\lambda \operatorname{dist}\left(x_{0}, x\right)} .
$$

This completes the proof.

\section{C.1 Proof of Proposition 6.2}

Proposition 6.2 which concerns the decay of the factional moments of the Green function will be deduced from Proposition C.1 through rather general considerations relating that quantity to to the eigenfunction correlators.

Lemma C.2. Let $H$ be a self-adjoint operator with purely discrete spectrum with eigenvalues $E_{1} \leq E_{2} \leq \ldots$ (counting multiplicity) and corresponding orthonormal set $\left\{\psi_{E_{n}}\right\}$ of eigenfunctions. Then:

1. For any pair of bounded intervals $I, J \subset \mathbb{R}$, and $s \in(0,1)$,

$$
\int_{I}\left|\left\langle\delta_{x}, P_{J}(H)(H-E)^{-1} \delta_{y}\right\rangle\right|^{s} d E \leq C_{s}(I)\left(\sum_{E_{n} \in J}\left|\psi_{E_{n}}(x)\right|\left|\psi_{E_{n}}(y)\right|\right)^{s},
$$

where $C_{s}(I):=4 s \int_{\mathbb{R}} t^{s-1} \min \left\{|I|, t^{-1}\right\} d t<\infty$.

2. For any Borel $J \subset \mathbb{R}$ and $E \in J$ with $\operatorname{dist}(E, \partial J)>0$ one has

$$
\begin{aligned}
& \left|\left\langle\delta_{x}, P_{J^{c}}(H)(H-E)^{-1} \delta_{y}\right\rangle\right|^{2} \\
& \quad \leq \sum_{\substack{m \in \mathbb{Z}: \\
I_{m} \cap J=\emptyset}} \frac{1}{\operatorname{dist}\left(E, I_{m}\right)^{2}} \sum_{E_{n} \in I_{m}}\left|\psi_{E_{n}}(x)\right|\left|\psi_{E_{n}}(y)\right|
\end{aligned}
$$

with $I_{m}:=(m, m+1]$.

Proof. Abbreviating $g(E):=\left\langle\delta_{x}, P_{J}(H)(H-E)^{-1} \delta_{y}\right\rangle$, we start from the representation

$$
\int_{I}|g(E)|^{s} d E=s \int_{0}^{\infty} t^{s-1}|\{E \in I:|g(E)|>t\}| d t .
$$


In terms of the spectral representation: $g(E)=\sum_{E_{n} \in J}\left(E_{n}-E\right)^{-1} \psi_{E_{n}}(x) \overline{\psi_{E_{n}}(y)}$. After a decomposition of $\psi_{E_{n}}(x) \overline{\psi_{E_{n}}(y)}$ into four terms corresponding to positive, respectively non-positive, real and imaginary parts, one may apply Boole's lemma [Bo57] to obtain:

$$
|\{E \in I:|g(E)|>t\}| \leq 4 \min \left\{|I|, t^{-1} \sum_{E_{n} \in J}\left|\psi_{E_{n}}(x)\right|\left|\psi_{E_{n}}(y)\right|\right\} .
$$

Substitution in C.12 yields the first assertion.

For a proof of the second bound we use the spectral decomposition and estimate

$$
\begin{aligned}
& \left|\left\langle\delta_{x}, P_{J^{c}}(H)(H-E)^{-1} \delta_{y}\right\rangle\right| \leq \sum_{E_{n} \in J^{c}} \frac{\left|\psi_{E_{n}}(x)\right| \mid \psi_{E_{n}}(y)}{\left|E_{n}-E\right|} \\
& \leq\left(\sum_{E_{n} \in J^{c}} \frac{\left|\psi_{E_{n}}(x)\right| \mid \psi_{E_{n}}(y)}{\left|E_{n}-E\right|^{2}}\right)^{1 / 2}\left(\sum_{E_{n}}\left|\psi_{E_{n}}(x)\right|\left|\psi_{E_{n}}(y)\right|\right)^{1 / 2}
\end{aligned}
$$

Using the Cauchy-Schwarz inequality and the fact that $\sum_{E_{n}}\left|\psi_{E_{n}}(x)\right|^{2}=1$, the last term is seen to be bounded by one. The claim then follows by decomposing the set $J^{c}$ into a the union of sets $I_{m} \cap J^{c}$ and estimating the denominator in the first factor on each of those sets.

Proof of Proposition 6.2 We pick $J \supset I$ such that $\operatorname{dist}(\partial J, \partial I)>0$ and decompose the Green function into the two parts according to the previous lemma. The estimates presented there yield the bound

$$
\begin{aligned}
& \int_{I} \mathbb{E}\left[\left|\left\langle\delta_{x},\left(H_{\mathcal{G}, \mathcal{B}}-E\right)^{-1} \delta_{y}\right\rangle\right|^{s}\right] d E \\
& \leq C \sup _{m \in \mathbb{Z}}\left(\mathbb{E}\left[\sum_{E_{n} \in(m, m+1]}\left|\psi_{E_{n}}(x)\right|\left|\psi_{E_{n}}(y)\right|\right]\right)^{s / 2}
\end{aligned}
$$

with the constant $C$ depending on $I$ and $s$. Thus Proposition 6.2 can be deduced from Proposition C.1

It may be noted that a converse relation also holds - the expectation in the righthand side of C.15 can be bounded in terms of a suitably averaged fractional moment of the Green function [Ai94].

\section{Discussion}

Our main result, Theorem 1.1 shows that regular tree graphs do not provide an example of the expected relation between the presence of ac spectrum for the infinite graph and random-matrix like statistics in the spectra of the corresponding finite volume restrictions. Let us therefore comment on a number of other directions in which it is 
natural to look for examples of such a relation.

As we saw, the negative result concerning the above relation reflects the fact that a finite tree is mostly surface. By implication, bulk averages of local quantities yield results representing the local mean not at sites deep within a tree but at sites near the canopy. In physicists discussions, the term 'Bethe lattice average' is intended to reflect the average at sites deep within the tree, and a standard device is used for extracting it from the bulk sum. For an extensive quantity such as $F_{L}=\operatorname{Tr} F\left(H_{\mathcal{H}_{L}}\right)$ where $\mathcal{H}$ denotes the homogeneous tree in which also the root has $K+1$ neighbors, the 'Bethe lattice average' $\langle F\rangle_{B L}$ is obtained not by taking $\lim _{L \rightarrow \infty} F_{L} /\left|\mathcal{H}_{L}\right|$, which gives the weighted canopy average (1.10), but rather (as in [MD93]) through the limit:

$$
\langle F\rangle_{B L}=\lim _{L \rightarrow \infty}\left(F_{L}-K F_{L-1}\right) / 2
$$

It would be of interest to see an adaptation of this approach for some separation of the statistics of eigenvalues corresponding to regions deep within the tree from the canopy average. However, even for the mean density of states, averaged over the disorder, it remains to be shown that the corresponding limit exists, and is given by a positive spectral measure. Furthermore, it is not clear how to use an analog of (D.1) for specific realizations of an operator with disorder, as the latter ruins the homogeneity.

Alternatively, one may look for graphs which have local tree structure without an obvious surface. Let us briefly comment on results which relate to two such cases: the random regular and the random Erdős-Rényi graph also known as the sparse random matrix ensemble.

The ensemble of random $c$-regular graphs [Bo85] consists of the uniform probability measure on graphs on $N \in \mathbb{N}$ vertices where each vertex has $c$ neighbors. It is known that as $N \rightarrow \infty$ the girth of the graph (the minimal loop length) diverges in probability and numerical simulations suggest [JM+99] that for large $c$ the eigenvalue spacing distribution of the adjacency operator approaches that of the Gaussian Orthogonal Ensemble (GOE).

The ensemble of Erdős-Rényi graphs results from the complete graph on $N \in \mathbb{N}$ vertices by removing bonds with probability $1-p$. This ensemble is known to have a percolation transition with an infinite tree-like connected component appearing as $N \rightarrow \infty$ if the average connectivity $c:=p N$ is bigger than one. The adjacency operator on these graphs is believed to exhibit a quantum percolation transition, i.e., the existence of extended eigenstates, at some value $c>1$. Numerical [Ev92, EE92, [BG00] and theoretical-physics calculations [MF91] suggest that the eigenvalue spacing distribution of the adjacency operator approaches GOE at least for large values of $c$ (possibly depending on $N$ ).

Since the graphs in both ensembles do not show a an obvious surface for finite $N$, they may offer a natural setting for the study of the relation between the extendedness of eigenstates of a finite volume random Schrödinger operator and its level statistics (a point which was also made in private discussions by T. Spencer). 


\section{Acknowledgement}

It is a pleasure to thank R. Sims and D. Jacobson for stimulating discussions of related topics. We also thank the referee for useful references concerning the appearance of the canopy graph in other studies. Some of the work was done at the Weizmann Institute (MA), at the Department of Physics of Complex Systems, and at University of Erlangen-Nürnberg (SW), Department of Physics. We are grateful for the hospitality enjoyed there. This work was supported in part by the NSF Grant DMS-0602360 and the Deutsche Forschungsgemeinschaft (Wa 1699/1).

\section{References}

[AK92] V. Acosta and A. Klein. Analyticity of the density of states in the Anderson model on the Bethe lattice. J. Stat. Phys. 69:277-305, 1992.

[AS93] T. Adachi and T. Sunada. Density of states in spectral geometry. Comment. Math. Helv. 68:480-493, 1993.

[AM93] M. Aizenman and S. Molchanov. Localization at large disorder and at extreme energies: an elementary derivation. Commun. Math. Phys., 157:245-278, 1993.

[Ai94] M. Aizenman. Localization at weak disorder: some elementary bounds. Rev. Math. Phys., 6:1163-1182, 1994.

[ASW06a] M. Aizenman, R. Sims and S. Warzel. Stability of the absolutely continuous spectrum of random Schrödinger operators on tree graphs. Prob. Theor. Rel. Fields. 136:363-394, 2006.

[ASW06b] M. Aizenman, R. Sims and S. Warzel. Absolutely continuous spectra of quantum tree graphs with weak disorder. Comm. Math. Phys., 264:371389, 2006.

[AF00] C. Allard and R. Froese. A Mourre estimate for a Schrödinger operator on a binary tree. Rev. Math. Phys., 12:1655-1667, 2000.

[AS86] B. Altshuler and B. I. Shklovski. Repulsion of energy levels and conductivity of metal samples. Sov. Phys. JETP, 64:127-135, 1986.

[BG00] M. Bauer and O. Golinelli. Random incidence matrices: moments of the spectral density. J. Stat. Phys., 103:301-337, 2000.

[Bo85] B. Bollobás. Random graphs. Academic, London, 1985.

[Bo57] G. Boole. On the comparison of transcendentals, with certain application to the theory of definite integrals. Philos. Trans. Royal Soc., 147: 780, 1857. 
[Br06] J. Breuer. Singular continuous and dense point spectrum for sparse trees with finite dimensions. Preprint math.SP/0608159.

[CKM87] R. Carmona, A. Klein, and F. Martinelli. Anderson localization for Bernoulli and other singular potentials. Commun. Math. Phys., 108:4166, 1987.

[CL90] R. Carmona and J. Lacroix. Spectral theory of random Schrödinger operators. Birkhäuser, Boston, 1990.

[D85] F. Delyon. Appearance of purely singular continuous spectrum in a class of random Schrödinger operators. J. Stat. Phys., 40:621-630, 1985.

[DKS83] F. Delyon, H. Kunz and B. Souillard. One-dimensional wave equations in disordered media. J. Phys. A: Math. Gen., 16:25-42, 1983.

[DS01] W. Dicks and T. Schick. The spectral measure of certain elements of the complex group ring of a wreath product. Geom. Dedicata 93:121-134, 2001.

[DR03] M. Disertori and V. Rivasseau. Random matrices and the Anderson model. Preprint math-ph/0310021

[EE92] S. N. Evangelou and E. N. Economou. Spectral density singularities, level statistics, and localization in a sparse random matrix ensemble. Phys. Rev. Lett. 68:361-364, 1992.

[Ev92] S. N. Evangelou. A numerical study of sparse random matrices. J. Stat. Phys., 69:361-364, 1992.

[Ef97] K. B. Efetov. Supersymmetry in disorder and chaos. Cambridge UP, Cambridge, 1997.

[FHS07] R. Froese, D. Hasler, and W. Spitzer. Absolutely continuous spectrum for the Anderson model on a tree: a geometric proof of Klein's theorem. Commun. Math. Phys. 269:239-257, 2007.

[GV06] G. M. Graf and A. Vaghi. A remark on an estimate by Minami. Preprint math-ph/0604033.

[GZ01] R. I. Grigorchuk and A. Zuk. The lamplighter group as a group generated by a 2-state automaton and its spectrum. Geom. Dedicata 87:209-244, 2001.

[JM+99] D. Jacobson, S. D. Miller, I. Rivin, and Z. Rudnick. Eigenvalue spacing for regular graphs. In D. A. Hejhal et al, eds., Emerging applications in number theory, Spinger, 1999.

[Ka02] O. Kallenberg. Foundations of modern probability. Springer, New York, 2002. 
[K195] A. Klein. The Anderson metal-insulator transition on the Bethe lattice. In D. Iagolnitzer, ed., Proceedings of the XIth international congress on mathematical physics, Paris, France, July 18-23, 1994, International Press, Cambridge, MA, 1995.

[K198] A. Klein. Extended states in the Anderson model on the Bethe lattice. Adv. Math., 133:163-184, 1998.

[KS80] H. Kunz and B. Souillard. Sur le spectre des operateurs aux difference finies aleatoire. Commun. Math. Phys., 78:201-246, 1980.

[KS99] T. Kottos and U. Smilansky. Periodic orbit theory and spectral statistics for quantum graphs. Ann. Phys. 274:76-124, 1999.

[KS06] R. Killip and M. Stoiciu. Eigenvalue Statistics for CMV Matrices: From Poisson to Clock via Circular Beta Ensembles. Preprint math-ph/0608002.

[MD93] J. D. Miller and B. Derrida. Weak disorder expansion for the Anderson model on a tree. J. Stat. Phys, 75:357-388, 1993.

[Mi96] N. Minami. Local fluctuation of the spectrum of a multidimensional Anderson tight binding model. Comm. Math. Phys. 177:709-725, 1996.

[MF91] A. D. Mirlin and Y. V. Fyodorov. Universality of the level correlation function of sparse random matrices. J. Phys. A: Math. Gen., 24:22732286, 1991.

[Mo81] S. A. Molchanov, The local structure of the spectrum of the onedimensional Schrödinger operator. Commun. Math. Phys., 78:429-446, 1981.

[PF92] L. Pastur and A. Figotin. Spectra of random and almost-periodic operators. Springer-Verlag, Berlin, 1992.

[RSS94] R. del Rio, B. Simon and G. Stolz, Stability of spectral types for SturmLiouville operators,. Math. Research. Lett., 1:437-450, 1994.

[Si96] B. Simon. Operators with singular continuous spectrum, IV: Graph Laplacians and Laplace-Beltrami operators. Proc. Amer. Math. Soc., 124:1177-1182, 1996.

[SW86] B. Simon and T. Wolff, Singular continuous spectrum under rank one perturbations and localization for random Hamiltonians. Commun. Pure Appl. Math., 39:75-90, 1986.

[Sh+93] B. I. Shklovskii, B. Shapiro, B. R. Sears, P. Lambrianides, and H. B. Shore. Statistics of spectra of disordered systems near the metal-insulator transition. Phys. Rev. B 47:11487-11490, 1993. 
[St01] P. Stollmann. Caught by disorder: bound states in random media. Birkhäuser, Boston, 2001.

[Sz89] A.-S. Sznitman. Lifshitz tail and Wiener sausage on hyperbolic space. Commun. Pure Appl. Math. 17:1033-1065, 1989.

[Sz90] A.-S. Sznitman. Lifshitz tail on hyperbolic space: Neumann conditions. Commun. Pure Appl. Math. 18:1-30, 1990.

[We81] F. Wegner. Bounds on the density of states in disordered systems. Z. Physik B, 44:9-15, 1981.

[Wo00] W. Woess. Random walks on infinite graphs and groups. Cambridge Tracts in Mathematics 138. Cambridge UP, Cambridge, 2000. 